ARTICLE

\title{
Prmt5 is a regulator of muscle stem cell expansion in adult mice
}

Ting Zhang ${ }^{1}$, Stefan Günther ${ }^{1}$, Mario Looso ${ }^{1}$, Carsten Künne ${ }^{1}$, Marcus Krüger ${ }^{1}$, Johnny Kim¹, Yonggang Zhou ${ }^{1}$ \& Thomas Braun ${ }^{1}$

Skeletal muscle stem cells (MuSC), also called satellite cells, are indispensable for maintenance and regeneration of adult skeletal muscles. Yet, a comprehensive picture of the regulatory events controlling the fate of MuSC is missing. Here, we determine the proteome of MuSC to design a loss-of-function screen, and identify 120 genes important for MuSC function including the arginine methyltransferase Prmt5. MuSC-specific inactivation of Prmt5 in adult mice prevents expansion of MuSC, abolishes long-term MuSC maintenance and abrogates skeletal muscle regeneration. Interestingly, Prmt5 is dispensable for proliferation and differentiation of $\mathrm{Pax}^{+}$myogenic progenitor cells during mouse embryonic development, indicating significant differences between embryonic and adult myogenesis. Mechanistic studies reveal that Prmt5 controls proliferation of adult MuSC by direct epigenetic silencing of the cell cycle inhibitor p21. We reason that Prmt5 generates a poised state that keeps MuSC in a standby mode, thus allowing rapid MuSC amplification under disease conditions.

\footnotetext{
${ }^{1}$ Department of Cardiac Development and Remodeling, Max-Planck Institute for Heart and Lung Research, Bad Nauheim 61231, Germany. Correspondence and requests for materials should be addressed to J.K. (email: Johnny.Kim@mpi-bn.mpg.de) or to Y.Z. (email: Yonggang.Zhou@mpi-bn.mpg.de) or to T.B. (email: Thomas.Braun@mpi-bn.mpg.de).
} 
O rgan-specific adult stem cells enable continuous regeneration of various tissues throughout adult life. Most adult stem cells are assumed to undergo constant turnover to ascertain self-renewal and tissue homeostasis, although dormant adult stem cells have been described in the haematopoietic system, which remain in a quiescent state for most of their lifetime and divide only rarely under severe stress conditions $^{1,2}$. Adult skeletal muscle stem cells (MuSC) are represented by a specialized subset of myofibre-associated cells called satellite cells and own a remarkable regenerative potential, which enables them to continuously replace myofibres by undergoing repeated rounds of activation and expansion under persisting disease conditions. Satellite cells originate during early embryonic development from a population of proliferating cells of the paraxial mesoderm. While most cells activate myogenic genes and form skeletal muscle fibres, some remain undifferentiated, adopt a satellite cell position in postnatal muscle and acquire a quiescent state ${ }^{3-5}$. After injury or excessive exercise, $\mathrm{Pax}^{+}$cells exit quiescence, proliferate and differentiate to generate new myofibres or fuse with existing myofibres, thereby fully restoring damaged skeletal muscle tissues $^{6-9}$. Several recent studies demonstrated that ablation of $\mathrm{Pax}^{+}$MuSCs prevents muscle repair under pathological conditions and during ageing (review in ref. 10). However, the molecular mechanisms that control satellite cell functions during skeletal muscle regeneration are only partially understood, although several factors directing the fate of MuSC have been identified (reviewed in refs 2,11).

To identify new regulators of MuSC activation, self-renewal, expansion and differentiation, we establish a systematic screening approach taking advantage of mass spectrometry-based protein profiling of FACS (fluorescence-activated cell sorting)-sorted MuSC $^{6,12,13}$ combined with short hairpin RNA (shRNA)mediated knockdown of MuSC-specific genes. Several genes are identified that strongly affect activities of MuSC in vitro, of which the histone arginine methyltransferase Prmt5 is chosen for indepth functional analysis. We find that Prmt5 is essential for adult MuSC proliferation and muscle regeneration by restricting p21 expression via direct epigenetic silencing, thereby allowing rapid expansion of MuSC. Since the lack of Prmt5 does not affect embryonic myogenesis, we postulate that prenatal muscle development and adult muscle regeneration use distinct genetic and epigenetic mechanisms for the control of muscle progenitor cell expansion.

\section{Results}

Identification of novel regulators controlling MuSC homeostasis. To determine the proteome of MuSCs, we isolated GFP-labelled stem cells (SCGFP) from skeletal muscles of $\operatorname{Pax}^{I C N} / Z E G$ mice ${ }^{6,14}$ via FACS (Supplementary Fig. 1a), which all expressed Pax7 protein and readily differentiated into myocytes (Supplementary Fig. 1b,c). Protein extracts of freshly isolated MuSCs were subjected to mass spectrometry analysis $(n=3)$ resulting in the identification of 135,341 peptides in all samples combined corresponding to 5,031 proteins in MuSC with at least one unique peptide (Supplementary Data 1). Notably, we detected numerous proteins known to be highly expressed in MuSCs including CD34, integrin $\alpha 7$, caveolin-1, Numb and $\beta 1$-integrin, but not haematopoietic or endothelial cell markers such as CD45 or CD31 (refs 12,13,15). Comparison with proteome data sets obtained from myofibres, $\mathrm{Pax}^{-}$mononuclear cells and the MuSC ${ }^{\text {PG }}$ fraction (satellite cells after percoll gradient and before FACS sorting) allowed us to identify 441 proteins that are exclusively present in MuSC but not in differentiated myofibres (Fig. $1 \mathrm{a}$ and Supplementary Data 2). Mass spectrometry data of randomly selected proteins (Wdr61, ABCC4, Lxn, Mustn1, P2RX4 and Prmt5) were validated by immunofluorescence staining of freshly isolated myofibres (Fig. 1b).

To analyse the function of MuSC-specific proteins, we generated a custom-arrayed lentiviral shRNA library (400 genes, one shRNA per well, on average five different shRNAs per gene; Fig. 1c and Supplementary Data 2). FACS-purified Pax Z ZsGreen MuSC $^{12}$ were transduced with shRNA expressing lentiviruses and analysed by high-throughput fluorescent microscopy $96 \mathrm{~h}$ post transduction for the ratio of $\mathrm{Pax}^{+}$versus total 4,6-diamidino-2-phenylindole $(\mathrm{DAPI})^{+}$cells (Fig. 1d), providing a read-out for genes affecting self-renewal, proliferation and differentiation of MuSC. shRNAs targeting Pax7 and Nf1 were included as quality controls ( $n=4$ wells for each plate). After selecting for genes, which yielded a strong shift of the ratio of $\mathrm{Pax}^{+}$versus total DAPI ${ }^{+}$cells after knockdown, we ended up with 30 genes inducing and 90 genes decreasing Pax7/DAPI ${ }^{+}$cell ratios after knockdown (Fig. 1e, Supplementary Fig. 1d and Supplementary Data 3).

Prmt5 is required for muscle regeneration. Next, we initiated a thorough analysis of the function of an exemplary candidate, the arginine methyltransferase Prmt5, that mediates H3R8 symmetric dimethylation (H3R8me2s; ref. 16). Prmt5 was recently implicated in the regulation of proliferation of embryonic stem cells ${ }^{17,18}$ and neural progenitor cells (NPCs) during brain development ${ }^{19}$, but its function in adult stem cells has remained elusive. Inactivation of Prmt5 using Prmt5 $5^{\text {loxP/loxP }}$ (Supplementary Fig. 2a) and Pax7CreERT2 mice ${ }^{6,7}$ (= MuSCspecific Prmt5 knockout mice, Prmt $5^{s K O}$ ) (Supplementary Fig. 2b) efficiently depleted Prmt5 mRNA in adult MuSC (Ctrl: $\quad 1.000 \pm 0.339, \quad n=3 ; \quad$ Prmt $^{s K O}: \quad 0.033 \pm 0.020, \quad n=4$; Supplementary Fig. 2c). Interestingly, tamoxifen (TAM)-treated Prmt5 ${ }^{5 K O}$ mice remained viable and displayed no obvious phenotype under physiological conditions 21 days after treatment compared with control animals ( $\mathrm{Ctrl}=$ Pax 7 CreERT2/ Prmt5 ${ }^{+/ l o x P}$; Supplementary Fig. 2d). No significant change of body weight (Ctrl $23.60 \pm 3.18 ; \mathrm{Prmt}^{\mathrm{sKO}} 22.67 \pm 2.52$, each $n=3$ ) and no morphological alterations of skeletal muscle tissue were apparent (Supplementary Fig. 2e f). Likewise, the number of satellite cells on sections of tibialis anterior (TA) muscle (Ctrl 20.33 $\pm 2.08 ;$ Prmt $^{s K O} 20.33 \pm 1.53$, each $n=3$ ) and freshly isolated flexor digitorum brevis (FDB) myofibres (Ctrl $30.00 \pm 3.61 ; P m t 5^{\text {sKO }} 30.00 \pm 2.00$, each $n=3$ ) did not differ between control and mutant littermates (Supplementary Fig. 2g,h). To investigate whether Prmt5-deficient satellite cells contribute to muscle regeneration, TA muscles of TAM-treated Prmt5 ${ }^{s K O}$ and control littermates were injected with cardiotoxin (CTX; Fig. 2a and Supplementary Fig. 2i). Strikingly, muscle regeneration was completely abolished in $\operatorname{Prm} 5^{\text {sKO }}$ mice at all investigated time points ( 7 and 14 days, and 4 months after injury). The virtually complete lack of regenerated muscle fibres (Fig. 2b and Supplementary Fig. 2j) was accompanied by a massive increase of fibrosis (Fig. $2 \mathrm{c}$ and Supplementary Fig. 2k). To analyse whether Prmt5 affects long-term satellite cell maintenance, we determined the number of MuSC 4 months after the initial TAM treatment. Importantly, we detected a significant decline of $\mathrm{Pax}^{+}$MuSC numbers both on cryosections from TA muscles (Fig. 2d; Ctrl 15.00 \pm 2.19 ; Prmt $^{5 K O} 5.17 \pm 2.32$, each $\left.n=6\right)$ and on freshly isolated myofibres from FDB muscle (Ctrl 23.00 \pm 2.65 ; Prmt $5^{\text {sKO }}$ $13.33 \pm 4.93$, each $n=3$; Fig. $2 \mathrm{e}$ ), indicating that Prmt5 is required for MuSC expansion during regeneration and needed to replenish the MuSC niche during physiological ageing. 
a

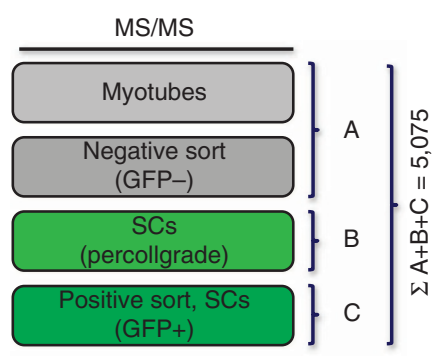

Proteome $\mathrm{SC}=(\mathrm{C}>\mathrm{B})-\Sigma \mathrm{A}=440$ b

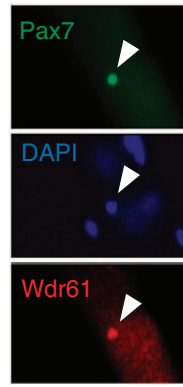

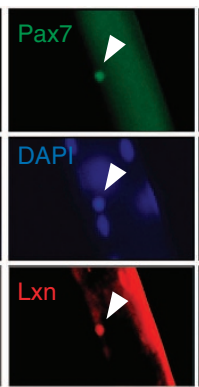
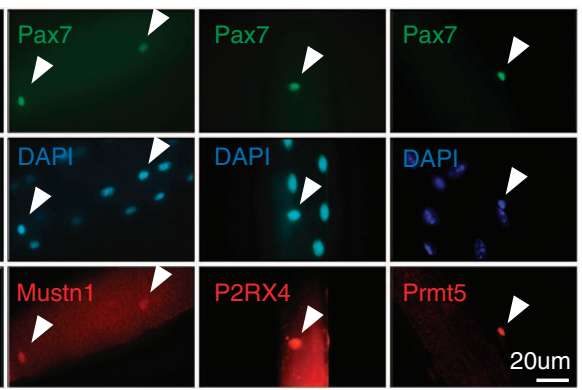

d

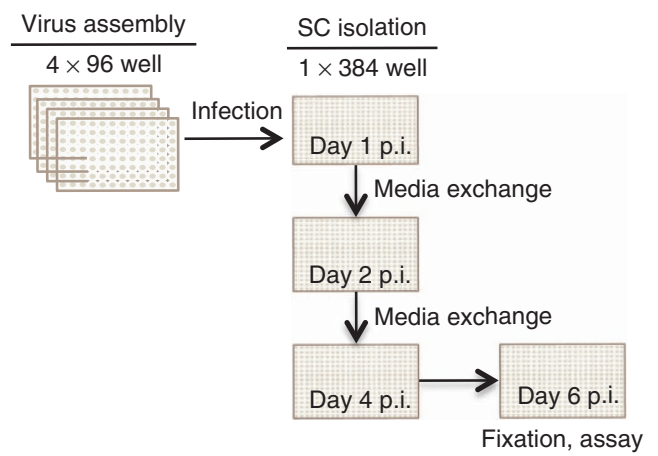

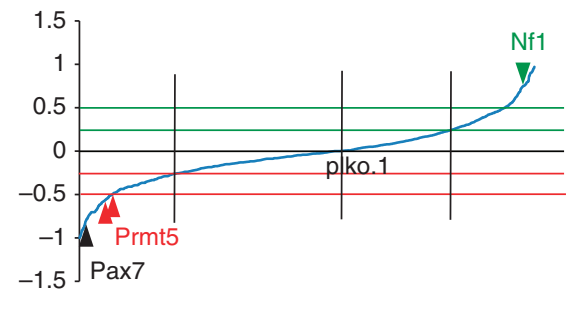

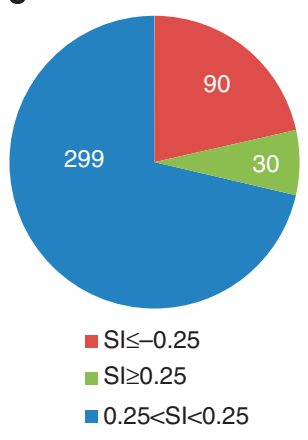

Figure 1 | Identification of regulators of stem cell homeostasis in a MuSC proteome-based shRNA screen. (a) Mass spectrometry (MS)-based identification of MuSC-enriched proteins (Proteome SC) using samples from fractionated skeletal muscles including purified myotubes, Pax7-GFPmononuclear cells, percoll-gradient purified and Pax7-GFP+ MuSC. (b) Immunofluorescence validation of identified proteins (red) counterstained with Pax7 antibody (green) and DAPI (blue; scale bar, $20 \mu \mathrm{m}$ ). (c) Schematic outline of the shRNA screen against corresponding genes of the satellite cell proteome. Phenotypic scores are calculated as ratios of Pax ${ }^{+} / \mathrm{DAPI}^{+}$nuclei for each well. (d) Poisson distribution of relative Pax7 expression for all 2,226 shRNAs targeting 419 genes. Red and green lines indicate phenotypic scores lower or higher than 0.25 percentiles normalized to plko.1 empty vector control. Knockdown of Pax7 reduces proliferation and enhances differentiation of MuSC ${ }^{6}$, whereas knockdown of Nf1 increases the numbers of Pax 7 expressing cells ${ }^{51}$. (e) shRNA knockdown identifies 90 and 30 candidate genes causing down- and upregulation of Pax $7^{+} / \mathrm{DAPI}^{+}$ratios, respectively. P.i., post infection; Pos, positive.

Prmt5 prevents depletion of the MuSC pool in $m d x$ mice. To further explore the role of Prmt5 in replenishing the MuSC pool, we utilized $m d x$ mice, which lack functional dystrophin resulting in continuous degeneration/regeneration of myofibres, accompanied by repeated activation and enhanced turnover of satellite cells $^{20}$. Treatment of 8 -week-old $\mathrm{Prmt5}^{\mathrm{sKO}} / \mathrm{mdx}$ compound mutant mice for 3 weeks with TAM (Fig. 3a) resulted in progressive loss of body weight, whereas Prmt5-deficient and $m d x$ mice gained weight similar to wild-type littermates (Fig. 3b,c). $\mathrm{Prmt}^{\mathrm{SKO}} / \mathrm{mdx}$ mice had a markedly lower body weight 4 months after initiation of the TAM treatment (Fig. 3b,c), and the diaphragm was markedly thinner compared with controls (Fig. 3d). Magnetic resonance imaging (MRI) measurements revealed a massive decrease of the total muscle mass normalized to tibia length of $\mathrm{Prmt}^{\mathrm{sKO}} / \mathrm{mdx}\left(87.5 \pm 27.2 \mathrm{~mm}^{3}\right.$ per $\left.\mathrm{mm}, n=4\right)$ but not of Prmt5 mutants $\left(211.5 \pm 10.0 \mathrm{~mm}^{3}\right.$ per $\left.\mathrm{mm}, n=3\right)$, wild-type mice $\left(224.9 \pm 32.3 \mathrm{~mm}^{3}\right.$ per $\left.\mathrm{mm}, n=5\right)$ and $m d x$ mice $\left(332.0 \pm 30.8 \mathrm{~mm}^{3}\right.$ per $\left.\mathrm{mm}, n=5\right)$, which gained muscle mass due to myofibre hypertrophy (Fig. 3e). Furthermore, Prmt $5^{s K O}$ $(4.67 \pm 2.52, n=3)$ and $P r m t 5^{s K O} / m d x$ mice $(2.80 \pm 2.49, n=5)$ displayed a significant reduction of Pax7 + MuSC at 6 months of age compared with controls (Ctrl 15.20 $\pm 2.39, n=5 ; m d x$ $32.67 \pm 18.72, n=3$; Fig. $3 f$ ). We concluded that Prmt 5 plays an essential role to maintain the MuSC pool and enables continuous muscle regeneration under chronic disease conditions.

Prmt5 controls proliferation and differentiation of MuSC. To investigate the cellular mechanisms responsible for the loss of the satellite cell pool and impaired muscle regeneration in Prmt5 $5^{\text {sKO }}$ mice, we first analysed FACS-purified MuSCs from Prmt5 ${ }^{5 K O}$ and control mice in vitro. Prmt5-deficient MuSCs showed a virtually complete arrest of cell proliferation as reflected by a marked reduction of the number of $\mathrm{Pax}^{+}, 5$-ethynyl-2' deoxyuridine (EdU)-incorporating cells (Ctrl 32.55 $\pm 5.94 \%$; Prmt $^{s K O} 8.29 \pm 4.24 \%$, each $n=6$ ), which is in line with the results from Prmt 5 knockdown experiments (Fig. 4a). Conversely, lentiviral overexpression of human Prmt5 stimulated proliferation of MuSC indicated by increased EdU incorporation (Ctrl ${ }^{G F P}$ $36.90 \pm 2.52 \%, n=8$; Prmt5 ${ }^{O E} 43.43 \pm 4.13 \%, n=6$; Fig. $\left.4 \mathrm{~b}\right)$. Moreover, we found a virtually complete absence of the formation of myogenic colonies on single myofibres from FDB muscles of TAM-treated Prmt $5^{\mathrm{sKO}}$ mice $(1.00 \pm 1.00, n=3)$ despite the presence of $\mathrm{Pax}^{+}$satellite cells (Fig. 4c). Genetic labelling of MuSC using a Rosa $26^{\text {nlac } Z}$ reporter in which removal of a stoplox cassette by $\operatorname{Pax} 7^{\mathrm{CreERT2}}$ resulted in activation of nlacZ expression uncovered a marked reduction of lacZ-positive MuSC in regenerating muscle of $P r m t 5^{s K O}$ mice 3 days after CTX injection (Ctrl 925 \pm 104 ; Prmt5 ${ }^{\text {sKO }} 212 \pm 6$, each $n=3$; Fig. 4d), indicating that Prmt5 is required for MuSC proliferation during the early phase of injury-induced muscle regeneration. Additional lineage tracing of MuSC using a Rosa26 ${ }^{Y F P}$ reporter revealed that Prmt5-deficient MuSC cells activated MyoD expression both on isolated myofibres and in single-cell cultures despite the failure to proliferate, suggesting that activation and proliferation of MuSC are not necessarily linked (Fig. 5a,b). However, activated, nonproliferative Prmt5-mutant MuSC failed to differentiate properly. 
a

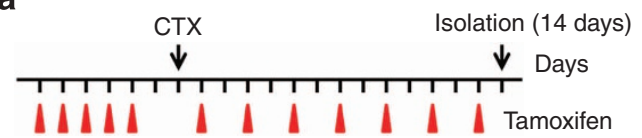

b
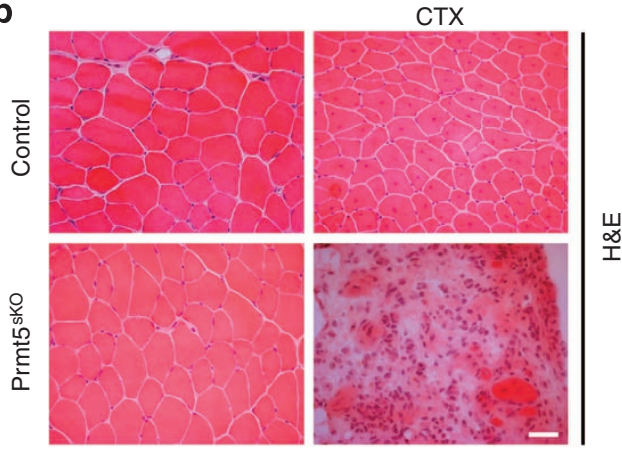

c
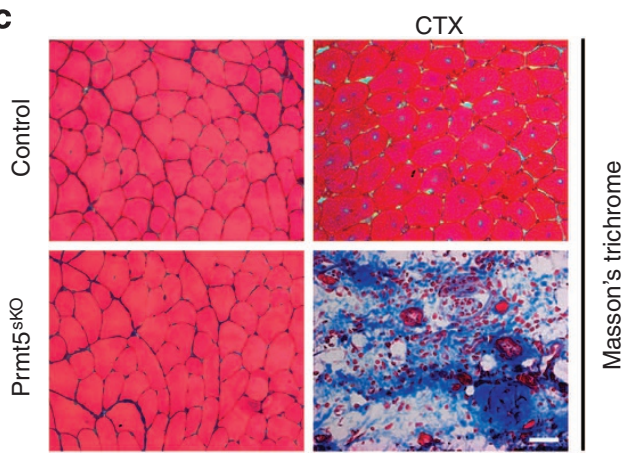

d
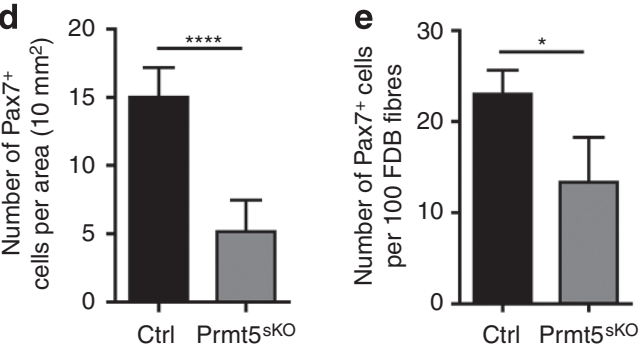

Figure 2 | Prmt5 is required for muscle regeneration and long-term maintenance of MuSC. (a) Schematic outline of CTX injection in TAMtreated control and Prmt5 $5^{5 K O}$ mutant littermates at the age of 8-9 weeks. (b) Haematoxylin and eosin (H\&E) and (c) Masson's Trichrome staining of injured or uninjured TA muscles of Prmt $5^{5 K O}$ mice and control littermates $(n=3$, each) 14 days after CTX injection. Scale bar, $20 \mu \mathrm{m}$. (d,e) Number of Pax $7^{+}$cells 4 months after TAM administration on TA cryosections $(n=6$, each; d) and freshly isolated FDB myofibres ( $n=3$, each; e) of Prmt $5^{5 K O}$ and control mice. The number of $\operatorname{Pax} 7^{+}$cell per $10 \mathrm{~mm}^{2}$ (d) or per 100 fibres (e) was counted. Error bars represent s.d.'s of the mean ( $t$-test: ${ }^{\star \star \star \star \star} P<0.0001,{ }^{\star} P<0.05 ; n=3$ ). Control (Ctrl): Pax7CreERT2 ${ }^{+/-/}$ Prmt5 +/loxP; Prm5 5KO: Pax7CreERT2+/-/Prmt5 loxP/loxP.

Expression of myogenin (MyoG), an early marker of muscle cell differentiation, was massively reduced in Prmt5 mutant MuSC attached to myofibres even after extended culture (Ctrl $27.17 \pm 2.25 \%$; Prmt $5^{\text {sKO }} 5.87 \pm 0.78 \%$, each $n=3$; Fig. $5 \mathrm{c}$ ). In addition, isolated MuSC from $\mathrm{Prmt}^{\mathrm{sKO}}$ mice expressed lower levels of MyoG (Ctrl 72.58 $\pm 5.30 \%$; Prmt $5^{s K O} 11.13 \pm 1.50 \%$, each $n=4)$ and did not form MF20 ${ }^{+}$myotubes efficiently (Ctrl $257.50 \pm 33.61 \mu \mathrm{m}^{2} ; \quad$ Prmt5 $^{\text {sKO }} 56.53 \pm 8.27 \mu \mathrm{m}^{2}$, each $n=3$; Fig. 5d,e). To further investigate the role of Prmt5 for myogenic differentiation, we inactivated the Prmt5 gene in vitro by treatment with 4-hydroxy-TAM (4-OH) (Fig. 6a) after amplification of isolated Prmt5 $5^{\text {sKO }}$ MuSC and induction of differentiation. Although we observed expression of the early differentiation marker MyoG in this experimental setting (Fig. 6b), differentiation of MuSC into MF20 ${ }^{+}$myotubes was essentially abrogated (Fig. 6c). We concluded that Prmt5 plays an additional role at a late stage of myogenic differentiation, independent of its function in proliferation and regulation of MyoG expression. Intriguingly, we also detected an increase of apoptosis in isolated Prmt $5^{\text {sKO }}$ MuSCs after induction of differentiation (Ctrl $0.27 \pm 0.12 \%$; Prmt $^{\text {sKO }} 9.00 \pm 1.75 \%$, each $\left.n=3\right)$, but not under conditions stimulating proliferation (Ctrl $0.08 \pm 0.07 \%$; $P r m t 5^{s K O}$ $0.11 \pm 0.11 \%$, each $n=3$; Fig. $6 \mathrm{~d}$ ), suggesting that either Prmt5 promotes cell survival during differentiation of MuSC or that lack of proliferation before differentiation favours apoptosis.

Prmt5 represses the cell cycle inhibitor p21 in MuSC. To gain a better mechanistic understanding of the action of Prmt5 in MuSC and to identify genes that might be directly regulated by Prmt5, we performed transcriptome analysis in 4-OH-treated MuSC from control and Prmt $5^{s K O}$ mice by RNA-sequencing (RNA-seq; Supplementary Data 4). Gene ontology (GO)-term analysis revealed an up- or downregulation of $\sim 500$ genes (false discovery rate $<0.05)$ involved in cell cycle control, DNA metabolism and replication after inactivation of Prmt5 (Supplementary Fig. 3a,b), which is consistent with the proliferation defects observed in Prmt5-deficient MuSC ${ }^{17,21}$. The upregulation of the cell cycle inhibitor p21 attracted our particular attention ${ }^{22-24}$. qRT-PCR analysis of freshly isolated FACS-sorted MuSC confirmed a strong upregulation of $p 21$ expression in Prmt5-deficient MuSCs indicating a transcriptional inhibition of $p 21$ by Prmt5 (Fig. 7a). In addition, we detected a clear upregulation of $p 21$ in $4-\mathrm{OH}-$ treated MuSC from Prmt5 $5^{\text {sKO }}$ mice (Fig. 7b) together with downregulation of CyclinB1, a p21 target gene, while transcription of myogenic factors including $\operatorname{Pax} 7, M y o D$ and Myf5 was not altered (Fig. 7b). To investigate a potential direct repression of the $p 21$ gene by Prmt5, we performed chromatin immunoprecipitation (ChIP) assays concentrating on four wellcharacterized regulatory regions of the murine $p 21$ gene: upstream enhancer like region (En), p53 binding site (p53BS), transcriptional start site (TSS) and downstream intronic CpG island (CpG; Fig. 7c; refs 25,26). In control MuSC, Prmt5 was highly enriched at the En and p53BS but not at the TSS and CpG sites, which was lost after treatment of $\operatorname{Prmt}^{5 K O} \mathrm{MuSC}$ with 4$\mathrm{OH}$ (Fig. 7d). Loss of Prmt5 binding caused a significant reduction of H3R8me2s at the p53BS site in Prmt5-deficient MuSCs (Fig. 7e), a significant loss of nucleosome occupancy at the TSS site (Fig. 7f) and increased H3K4 trimethylation at the CpG site (Fig. $7 \mathrm{~g}$ ), which is all consistent with suppression of $p 21$ by Prmt5. Binding of Prmt5 to the p53BS prompted us to ask whether Prmt5 suppresses p21 expression by preventing recruitment of $\mathrm{p} 53$. Surprisingly, inactivation of Prmt5 prevented binding of $\mathrm{p} 53$ to the p53BS in the $p 21$ locus, thereby suggesting $\mathrm{p} 53$-independent upregulation of $p 21$ in Prmt5-deficient MuSCs (Fig. 7h). This conclusion was also supported by normal p53 mRNA and protein levels in Prmt5 mutant compared with control MuSCs, although we detected accumulation of Mdm4 splicing variants that were shown to stabilize p53 in Prmt5-deficient NPCs (Fig. 7i,j; ref. 19).

To investigate whether impaired proliferation of Prmt5-mutant MuSC is mediated by $\mathrm{p} 21$, we generated $P r m t 5^{5 \mathrm{KO}} / \mathrm{p} 21^{-1-}$ compound mutant mice ${ }^{22}$. Intriguingly, we observed a significant increase of proliferation of MuSC isolated from Prmt $5^{s K O}$, $p 21^{-1-}(25.25 \pm 1.47 \%, n=3)$ compared with $P r m t 5^{s K O}$ mice (11.34 $\pm 1.50 \%, n=3$; Fig. 8a). Furthermore, we found an increase of the number of myogenic colonies on 3-day cultured 
a

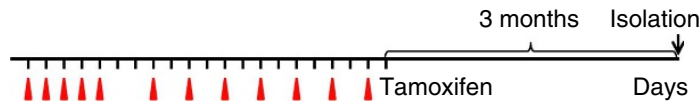

b
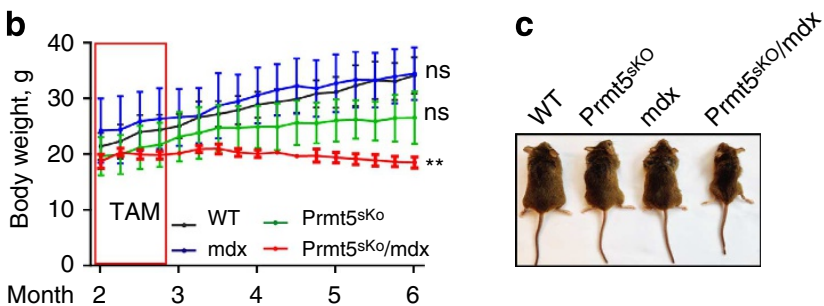

e

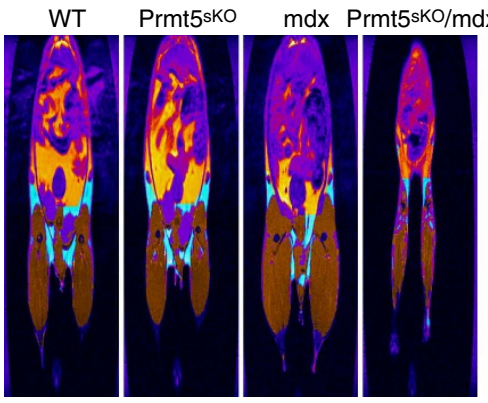

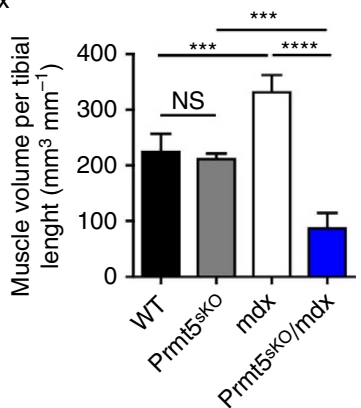

d

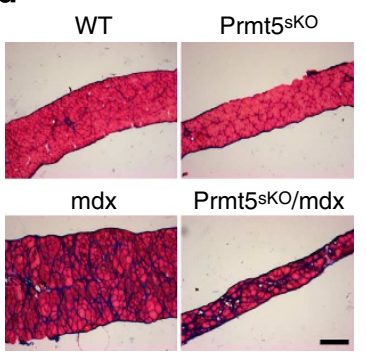

$\mathbf{f}$

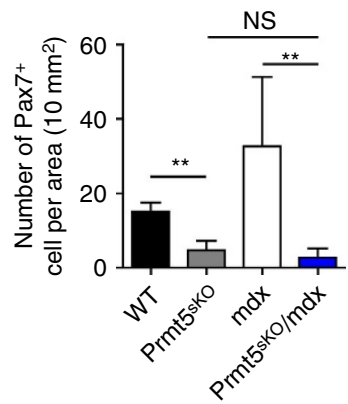

Figure 3 | Continuous muscle regeneration depletes the MuSC pool of Prmt5 $5^{\text {sKo }}$ mice. (a) Schematic outline of the TAM administration and analysis of wild-type (WT), Prmt5 $5^{5 K O}, m d x$ and Prmt5 $5^{5 K O} / m d x$ mice $(n=3$, each). (b-d) Prmt5 $5 \mathrm{KO} / \mathrm{mdx}$ mice $(n=3)$ show lower body weight $(\mathbf{b}, \mathbf{c})$ and thinner diaphragm (d) compared with WT $(n=3), \mathrm{mdx}(n=3)$ and $\operatorname{Prmt5}^{5 K O}(n=3)$ littermates 3 months after TAM administration. Scale bar, $100 \mu \mathrm{m}(\mathbf{d})$. (e) MRI measurements of decreased and increased muscle volume (brown colour) in Prmt $5^{5 K O} / \mathrm{mdx}$ and $\mathrm{mdx}$ mice, respectively, 3 months after TAM administration (WT and $m d x, n=5$ each; Prmt $5^{5 K O}, n=3 ; P r m t 5^{5 K O} / m d x, n=4$ ). Quantification of muscle volume normalized to tibia length is shown on the right. Error bars represent s.d.'s of the mean ( $t$-test: ${ }^{\star \star \star \star} P<0.0001$; ${ }^{\star \star \star} P<0.001 ; N S, P>0.05$ ). (f) Decreased numbers of Pax ${ }^{+}$cells in TA muscles of Prmt $5^{5 K O} / m d x$ mice $(n=5)$ compared with WT $\left(n=5\right.$ and $m d x(n=3)$ littermates. The number of Pax $7^{+}$cells per $10 \mathrm{~mm}^{2}$ area was counted on cryosections. Error bars represent s.d.'s of the mean ( $t$-test: $\left.{ }^{\star \star} P<0.01 ; N S, P>0.05\right)$. Control (Ctrl): Pax 7 CreERT2 $2^{+/}-/ P r m t 5^{+/ l o x P} ;$ Prm5 $5^{5 K O}$ : Pax7CreERT2 ${ }^{+/} / /$Prmt5 $5^{\text {loxP/loxP }}$. NS, not significant.

FDB myofibres in $\mathrm{Prmt}^{\mathrm{sKO}} / \mathrm{p} 21^{-1-}$ mice $(22.40 \pm 7.30 \%, n=5)$ compared with $\operatorname{Prmt5}^{\text {sKO }}$ mice $(6.00 \pm 2.16 \%, n=4$; Fig. $8 \mathrm{~b})$. However, inactivation of p21 in Prmt5 mutant mice failed to restore skeletal muscle regeneration, indicating that Prmt5 controls MuSC expansion and differentiation not exclusively by direct regulation of the $p 21$ gene (Fig. $8 c, d)$.

Prmt5 is dispensable for embryonic muscle development. It is widely assumed that embryonic and adult myogenesis are regulated by similar molecular cues, although a number of important differences are apparent ${ }^{10}$. Hence, we wanted to know whether Prmt 5 does not only control MuSC and muscle regeneration but is also involved in the formation of skeletal muscles during development, in particular, since Prmt5 has been claimed to regulate expression of Myf5, MyoD, Myogenin and Mef $2 c$ during zebrafish myogenesis ${ }^{27}$. Therefore, we deleted the Prmt5 gene in the myogenic lineage using the constitutively active Pax7Cre knock-in mouse strain $\left(P a x 7^{C r e}\right)^{14}$. qRT-PCR analysis of FACSsorted Pax $7^{Z s G r e e n}$ myogenic cells from Pax 7 Cre $/$ Prmt $55^{\text {loxP/loxP }}$ mutant embryos (hereafter referred to as $P r m t 5^{m K O}$ ) verified efficient inactivation of Prmt5 expression in embryonic muscle progenitor cells (data not shown). Analysis of control and Prmt5 ${ }^{m K O}$ mutant embryos at E9.5, E12.5 and E16.5 revealed no obvious defects in skeletal muscle formation (Fig. 9a). The normal presence of $\mathrm{Pax}^{+}, \mathrm{MyoG}^{+}$and $\mathrm{MF}^{+} 0^{+}$cells in embryonic forelimbs muscle of E12.5 and E14.5 Prmt5 $5^{\mathrm{mKO}}$ embryos (Fig. 9b,c) suggested that loss of Prmt5 in Pax7 ${ }^{+}$myogenic progenitor cells neither affects their expansion nor differentiation during embryonic muscle development. Similarly, lack of Prmt5 had no effects on Pax7, MyoD and MyoG expression in forelimb and hindlimb muscles at E16.5 when $\mathrm{Pax} 7^{+}$muscle progenitor cells play an essential role for fetal muscle growth ${ }^{28}$ or on prenatal muscle growth until birth (Fig. 9d,e). Despite the absence of an apparent skeletal muscle phenotype, most Prmt $5^{\mathrm{mKO}}$ mutants died around birth, which we attributed to the activity of Pax 7 Cre and consecutive loss of Prmt5 in the central nervous system $(\mathrm{CNS})^{19}$.

\section{Discussion}

Our screen identified several novel potential regulators together with molecules that have already been documented to control the fate of MuSC. Prominent examples include Smad3 (ref. 29) and syndecan-4 (ref. 30). We also identified several epigenetic modifiers including $\mathrm{Wdr} 91$, the poly (ADP-ribose) polymerase Parp12 and Ash2l, a component of the Mll2 complex that mediates H3K4 methylation, which has been shown to form a complex with the transcription factor Pax7 to regulate Myf5 expression and satellite cell proliferation ${ }^{31,32}$. The histone arginine methyltransferase Prmt5 attracted our particular attention also because we identified several known interaction partners of Prmt5 in the screen including Myd88 (ref. 33) and Mapk13 (also known as p38delta), a component of the mitogenactivated protein (MAP) kinase pathway ${ }^{34}$, suggesting that Prmt5-dependent mechanisms play a preeminent role in the regulation of MuSC proliferation and differentiation.

During adulthood MuSC mostly exist in a resting, quiescent state but must be able to expand rapidly in order to regenerate damaged muscle tissue. Relaxed control of quiescence might lead 
a
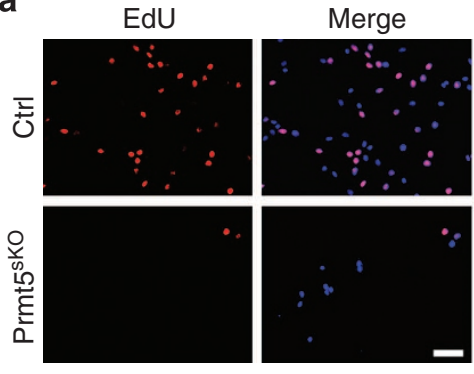

C
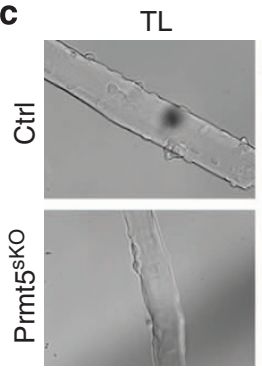

d

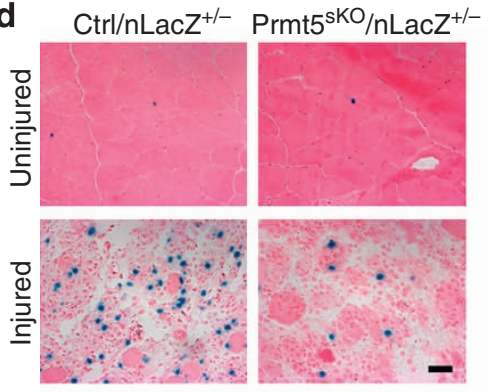

DAPI
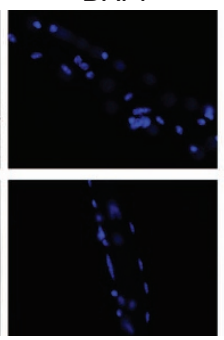

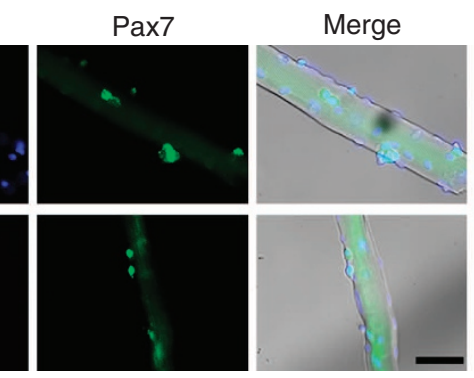

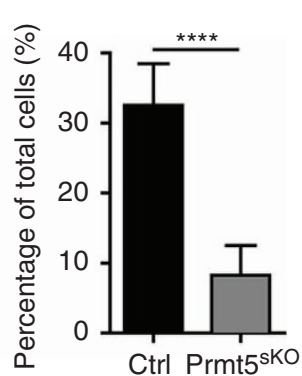

Ctrl Prmt5 sKo
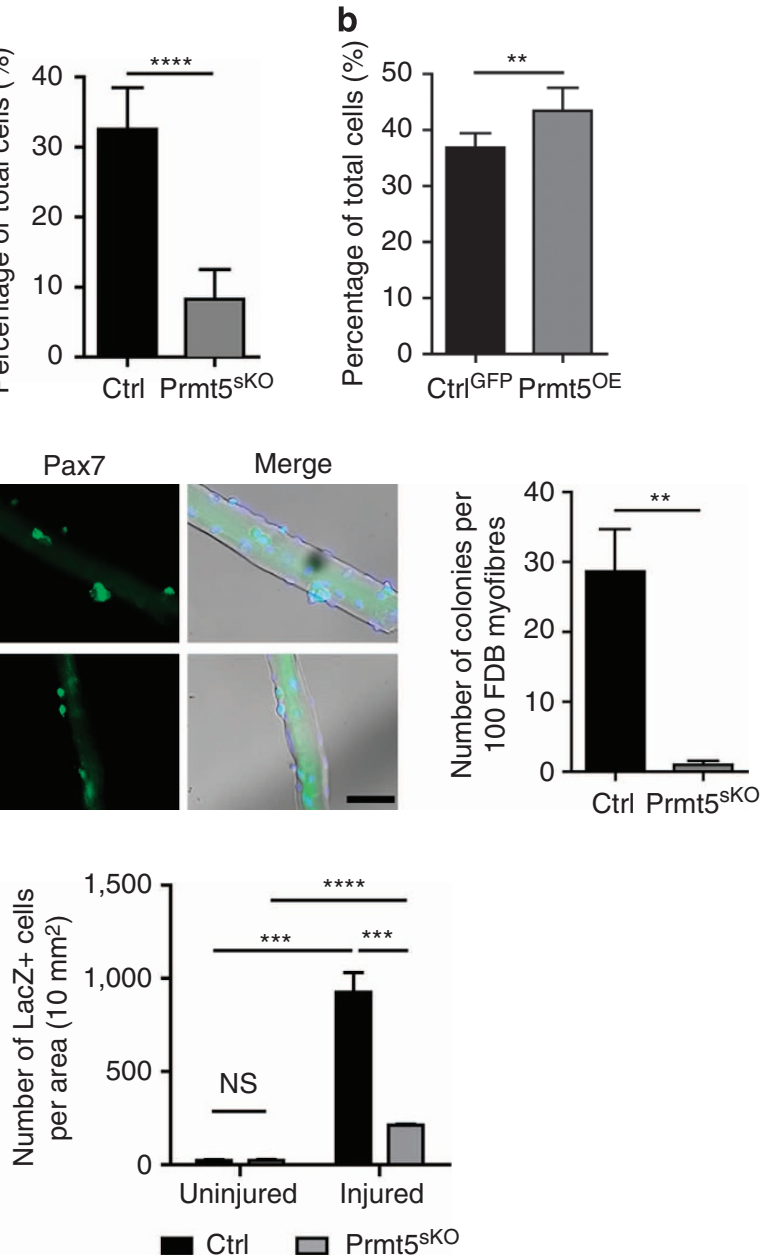

Figure 4 | Prmt5 is required for proliferation of MuSC. (a) Representative immunofluorescence images of cultured MuSC from control and Prmt $5^{5 K O}$ mice $\left(n=6\right.$, each) after EdU incorporation. Quantification of EdU ${ }^{+}$cells is shown on the right. Error bars represent s.d.'s of the mean ( $t$-test: $\left.{ }^{\star \star \star \star} P<0.0001\right)$. (b) Percentage of EdU +/MuSC relative to all Pax $7^{+}$cells after infection with lentiviruses overexpressing Prmt5 $(n=6)$ or GFP $(n=8)$. Error bars represent s.d.'s of the mean $\left(t\right.$-test: $\left.{ }^{\star \star} P<0.01\right)$. (c) Immunofluorescence analyses of Pax $7^{+}$-colonies on isolated FDB myofibres of control and Prmt5 $5^{5 K O}$ mice after 3-day culture $\left(n=3\right.$, each). Scale bar, $50 \mu \mathrm{m}$. Total numbers of Pax $7^{+}$colonies from 100 myofibres are counted. Quantifications are shown on the right. Error bars represent s.d.'s of the mean ( $t$-test: $\left.{ }^{\star \star} P<0.01\right)$. (d) Nuclear LacZ (nLacZ) staining for identification of MuSC in normal and regenerating TA muscle sections from Prmt5 ${ }^{\text {sKO }}$ mice and control littermates 3 days after CTX injection $(n=3$, each). Scale bar, $20 \mu \mathrm{m}$. The numbers of $\mathrm{LacZ}^{+}$MuSC per $10 \mathrm{~mm}^{2}$ section area are displayed. Error bars represent s.d.'s of the mean (t-test: ${ }^{\star \star \star \star} P<0.0001 ;{ }^{\star \star \star} P<0.001 ; \mathrm{NS}, P>0.05 ; n=3$ ). Control (Ctrl): Pax7CreERT2 ${ }^{+/-} /$Prmt5 $+/ /$loxP $;$Prmt5 $5^{\text {SKO }}$ : Pax7CreERT2 $+/-/$ Prmt5loxP/loxP. NS, not significant; TL, transmitted light.

to over-proliferation, depletion of the stem cell pool and might favour tumour formation. Failure to respond appropriately to proliferative cues will impair self-renewal of MuSC and compromise regeneration. Prmt5 seems to be a decisive component of the regulatory network that maintains this intricate balance and keeps MuSC in a poised standby mode (Fig. 10). In contrast, embryonic myogenesis is characterized by the rapid expansion of myogenic progenitor cells, which need to form skeletal muscles in a relatively short time period alleviating the need to enter a quiescent, non-proliferative state. Hence, it makes sense that the role of Prmt5 in the regulation of cell proliferation differs significantly between embryonic and adult myogenesis, whereas the control of muscle lineage determination and differentiation seems to follow a similar pattern ${ }^{10,35-37}$.

A major function of Prmt5 for conferring a reversible resting state to MuSC is apparently the restriction of p21 expression. Reduced expression of Prmt5 in MuSC will result in upregulation of $p 21$, which increases the threshold for cell cycle re-entry (Fig. 10). Although we detected Prmt5 by immunofluorescence in virtually all MuSC, its level of activity and hence regulation of $p 21$ might vary, thereby contributing to the heterogeneity of MuSC. MuSC with lower Prmt5 activity might constitute a reserve population that is only activated under severe stress conditions. Alternatively, differential regulation of Prmt5 activity in asymmetrically dividing MuSC might distinguish cells returning to quiescence from those that undergo rapid expansion. Careful quantitative evaluation of Prmt5 activity in single MuSC will solve these questions in the future.

We do not claim that the epigenetic repression of $p 21$ is the only mechanism by which Prmt5 arrests MuSC proliferation, in particular, since RNA-seq analysis identified several additional cell cycle regulators that might also be regulated by Prmt5 either directly or indirectly. Inactivation of p21 in Prmt5-deficient MuSC failed to rescue muscle regeneration fully, although proliferation of MuSC could be partially restored, indicating different modes of action of Prmt5 independent of p21. In fact, additional functions of Prmt5 in the regulation of progenitor cell behaviour have been reported previously. In embryonic stem (ES) cells, Prmt 5 promotes pluripotency by modulating the cytoplasmic LIF/Stat3 signalling pathway, indirectly suppressing genes 
a

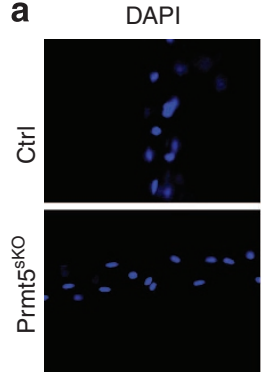

b
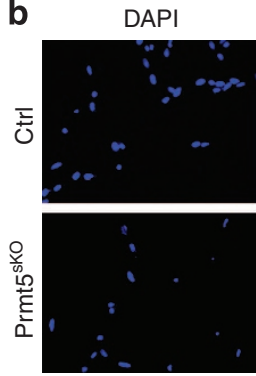

C
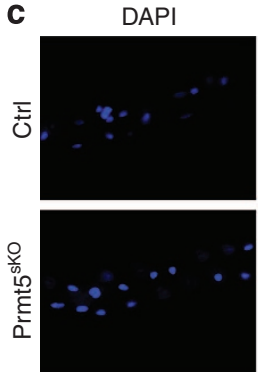

d DAPI
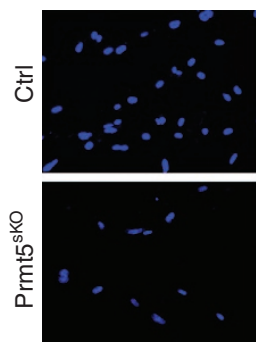

e
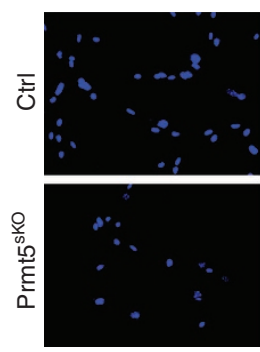

YFP

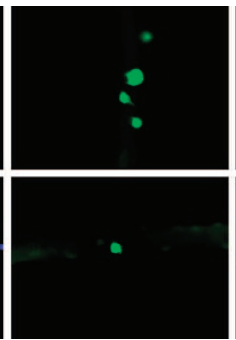

MyoD
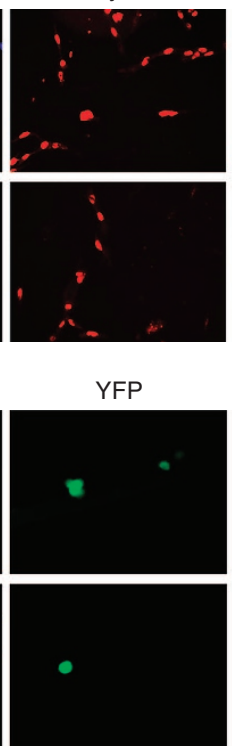

Myogenin
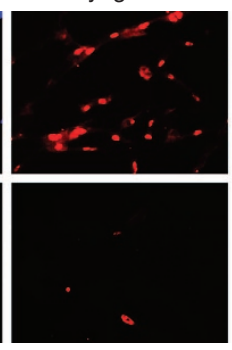

MF20
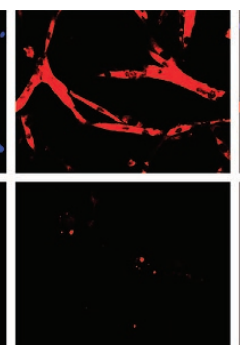

MyoD

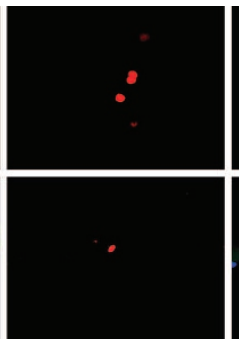

Merge

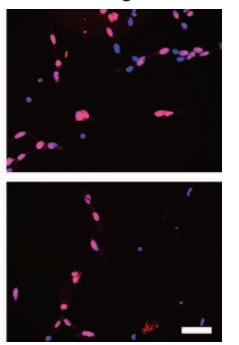

MyoG

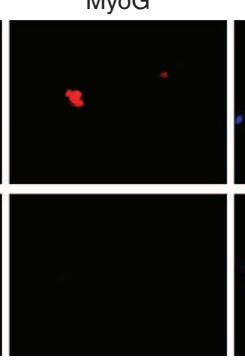

Merge

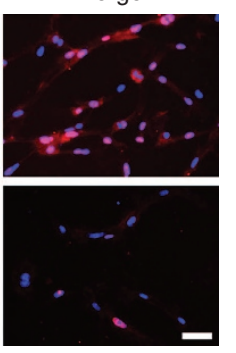

Merge
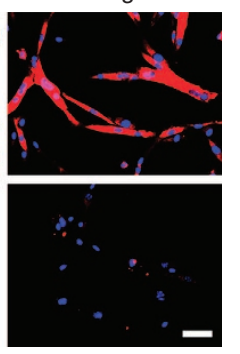

Merge
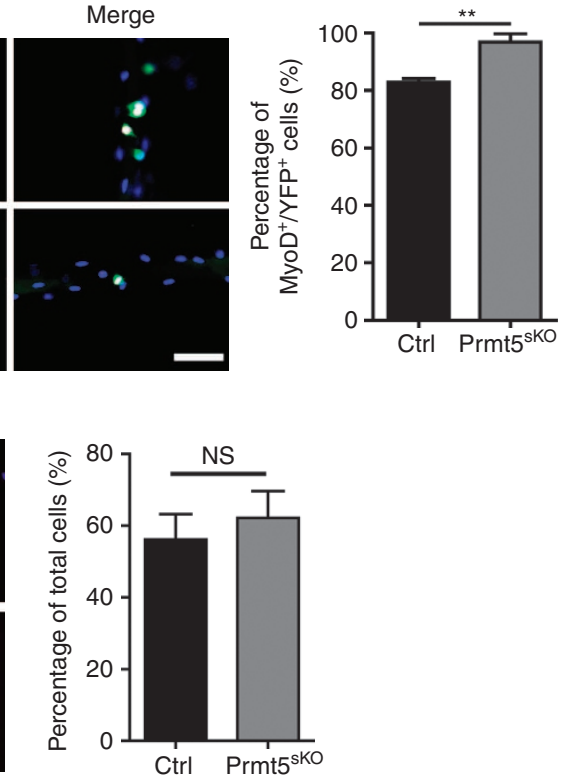

Ctrl Prmt5 ${ }^{\text {sKo }}$
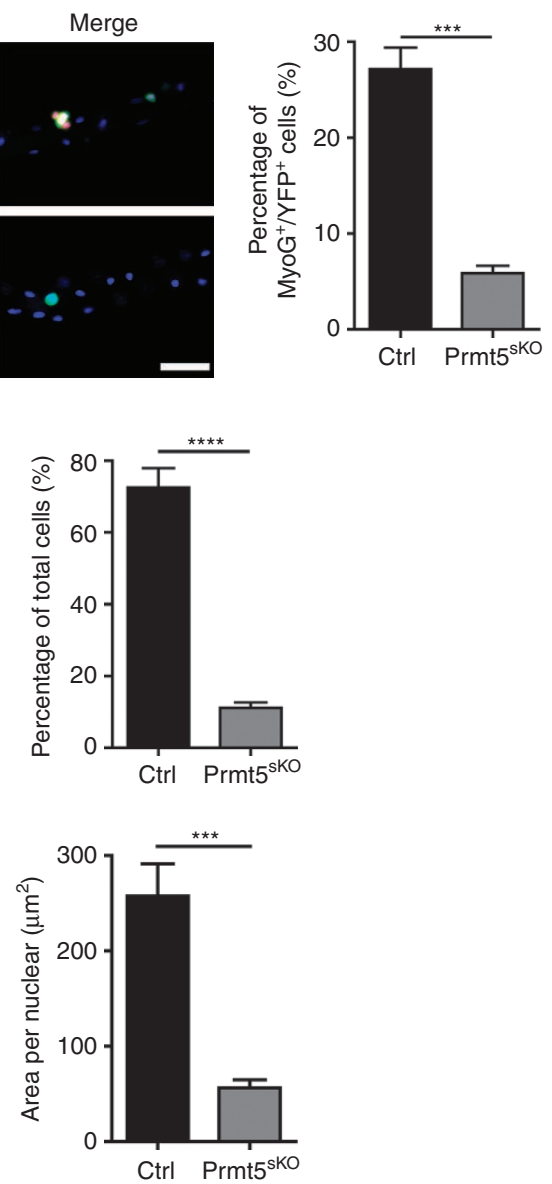

Figure 5 | Prmt5 is required for differentiation of MuSC. (a,c) Immunofluorescence analysis of double $\mathrm{YFP}^{+} / \mathrm{MyoD}^{+}$cells $(\mathbf{a})$ and double $\mathrm{YFP}^{+}$and $\mathrm{MyoG}^{+}$cells (c) on isolated FDB myofibres of control and Prmt5 $5^{5 \mathrm{KO}}$ mice after 3 -day culture $\left(n=3\right.$, each). Scale bar, $50 \mu \mathrm{m}$. Total numbers of Pax $7^{+}$ colonies from 100 myofibres are counted. Quantifications are shown on the right. Error bars represent s.d.'s of the mean $\left(t\right.$-test: $\left.{ }^{\star \star} P<0.01,{ }^{\star \star \star} P<0.001\right)$. (b) Immunofluorescence staining of FACS-purified YFP ${ }^{+}$cells cultured for 3 days in proliferation medium for MyoD ( $n=3$, each). Scale bar, $50 \mu$ m. The percentage of $\mathrm{MyoD}^{+}$cells is shown on the right. Error bars represent s.d.'s of the mean ( $t$-test: NS, $P>0.05$ ). (d) Immunofluorescence staining for MyoG of FACS-purified YFP + cells cultured for 3 days in proliferation medium followed by 2 days in differentiation medium $(n=4$, each). Scale bar, $50 \mu$ m. The percentage of $\mathrm{MyoG}^{+}$cells is shown on the right. Error bars represent s.d.'s of the mean ( $t$-test: $\left.{ }^{\star \star \star \star} P<0.0001\right)$. (e) Immunofluorescence images of

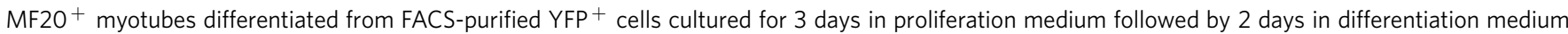
$\left(n=3\right.$, each). Scale bar, $50 \mu \mathrm{m}$. The area of MF20 ${ }^{+}$cells is shown on the right. Error bars represent s.d.'s of the mean $\left(t\right.$-test: $\left.{ }^{\star \star \star} P<0.001\right)$. Control $(C \operatorname{trl})$ : Pax7CreERT2 $2^{+/-} /$Prmt5 $^{+/ / \text {loxP }}$; Prmt5 ${ }^{\text {SKO}}$ : Pax7CreERT2 $2^{+/-} /$Prmt5 $^{\text {loxP/loxP }}$. 

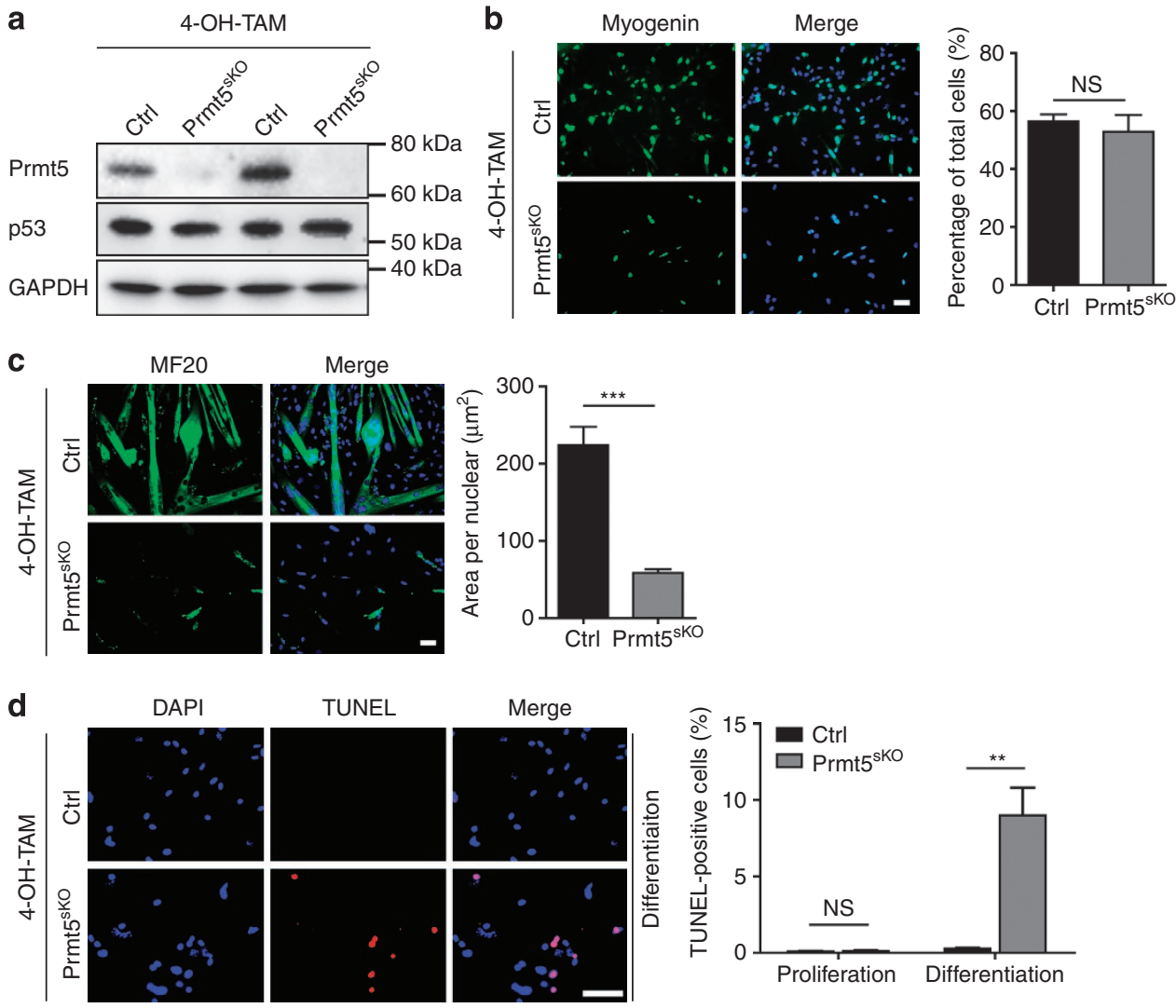

Figure 6 | Prmt5 is required for survival of MuSC upon differentiation. (a) Western blot analysis of Prmt5 and p53 protein levels in isolated MuSC of Prmt5 $5^{5 K O}$ and control littermates after in vitro 4-OH TAM treatment $(n=2)$. (b,c) Immunofluorescence staining for MyoG (b) and MF20 (c) of control and $\mathrm{Prmt}^{5 \mathrm{KO}}$ MuSC, which were in vitro amplified and subsequently treated with 4-OH-TAM, 2 days after induction of differentiation. Scale bars, $20 \mu \mathrm{m}$. The percentage of $\mathrm{MyoG}^{+}$and MF20 ${ }^{+}$cells is shown on the right. Error bars represent s.d.'s of the mean. $\left(t\right.$-test: $\left.{ }^{\star \star \star} P<0.001 ; \mathrm{NS}, P>0.05, n=3\right)$. (d) TUNEL assays of FACS-sorted control and Prmt5-deficient MuSC in differentiation medium ( $n=3$, each). Scale bar, $20 \mu \mathrm{m}$. Quantifications are shown on the right. Error bars represent s.d.'s of the mean ( $t$-test: $\left.{ }^{\star \star} P<0.01 ; \mathrm{NS}, P>0.05\right)$. Control (Ctrl): Pax $7 \mathrm{CreERT2}{ }^{+/}-/$Prmt5 ${ }^{+/ / 0 x P} ;$ Prm5 ${ }^{5 K O}$ : Pax7CreERT2 ${ }^{+/-} /$Prmt5 $5^{\text {loxP/loxP. }}$. NS, not significant.

that are associated with ES cell differentiation ${ }^{17}$. In NPCs, Prmt5 regulates alternative splicing of $\mathrm{Mdm} 4$ that in turn stabilizes p53, which causes upregulation of p21 and inhibition of cell cycle progression ${ }^{19}$. Superficially, the findings in NPC appear to partially recapitulate the situation in MuSC, but a more careful analysis reveals fundamental differences in the mode of action. Inactivation of Prmt5 in MuSC does not change the mRNA and protein level of $\mathrm{p} 53$, although alternative splicing of Mdm4 was altered. Furthermore, we found that binding of p53 to the $p 21$ locus was lost after inactivation of Prmt5, indicating that activation of p21 in Prmt5-deficient MuSC does not depend on $\mathrm{p} 53$.

Although suppression of MuSC expansion by upregulation of p21 dominated the phenotype of $\mathrm{Prmt}_{5} 5^{\mathrm{SKO}}$ mice, lineage-tracing experiments revealed that Prmt5-deficient MuSC failed to express myogenin, indicating that Prmt5 mutant MuSCs are unable to differentiate and form myofibres in vivo. This conclusion was also supported by the failure of MuSC to form myotubes even when Prmt5 was deleted after initiation of $M y o D$ expression, a phenomenon that was also observed in $\mathrm{C} 2 \mathrm{C} 12$ myoblasts $^{38}$. In addition, the timed inactivation of Prmt5 in differentiating MuSC suggests an additional role for terminal myogenic differentiation after expression of MyoG has commenced. Interestingly, induction of differentiation of Prmt5 mutant MuSC triggered apoptosis, which might be related to the differentiation block and contribute to the loss of MuSC during regeneration and ageing.
Our study revealed that inactivation of Prmt5 in MuSC of $m d x$ mice resulted in a severe loss of muscle volume and recapitulated several symptoms of human Duchenne muscular dystrophy within 90 days. The findings emphasize the pivotal role of MuSC in maintaining muscle mass under disease conditions. A similar phenotype was described recently using $m d x$ mice completely lacking telomerase activity $\left(m d x / m T R^{2 G} \text { mice }\right)^{20}$. However, in $m d x / m T R^{2 \mathrm{G}}$ mice, a massive atrophy of the diaphragm was only visible after 60 weeks, indicating that the lack of Prmt5 had more severe consequences in MuSC function than loss of telomerase activity. We believe that $P r m t 5^{s K O} / m d x$ mice might serve as a valuable model to study effects of therapeutic interventions on dystrophin-deficient myofibres without the interference of MuSC constantly replenishing lost or damaged myofibres.

Remarkably, muscle mass remained rather stable in Prmt $5^{5 K O}$ mice under physiological conditions for at least 3 months despite a significant decline of the number of MuSC and the failure of MuSC to expand. This finding allows two conclusions: (i) under physiological conditions, MuSC contribute only to a minor degree to the maintenance of muscle mass; and (ii) a significant proportion of MuSC undergoes self-renewal during a 3-month period. However, the second conclusion has to be viewed with caution, since it is possible that the lack of Prmt5 induces cell death of MuSC without prior activation and induction of proliferation, although we did not find evidence for such a scenario in our experiments. In the future, it will be interesting to further exploit the Prmt5 ${ }^{5 K O}$ model (Fig. 10) to study the role of 

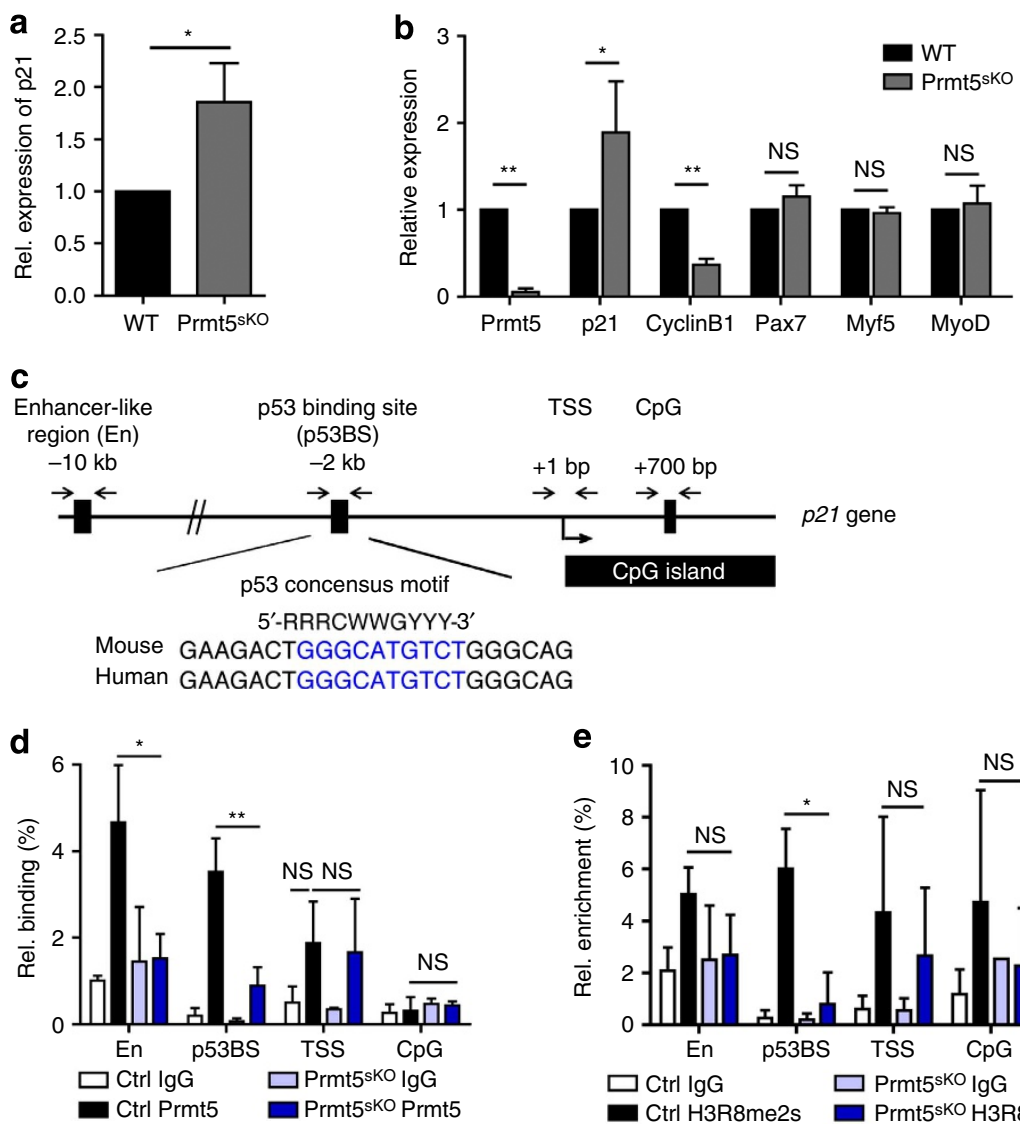

TSS CpG

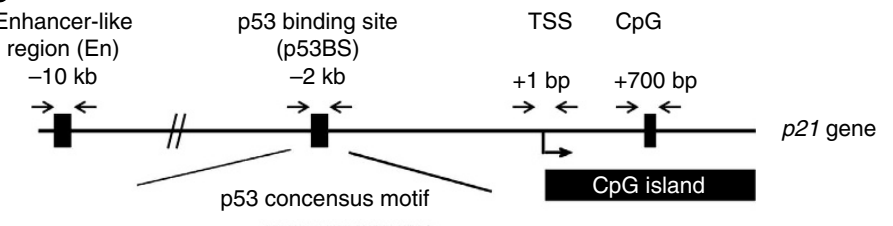

Mouse GAAGACTGGGCATGTCTGGGCAG

Human GAAGACTGGGCATGTCTGGGCAG
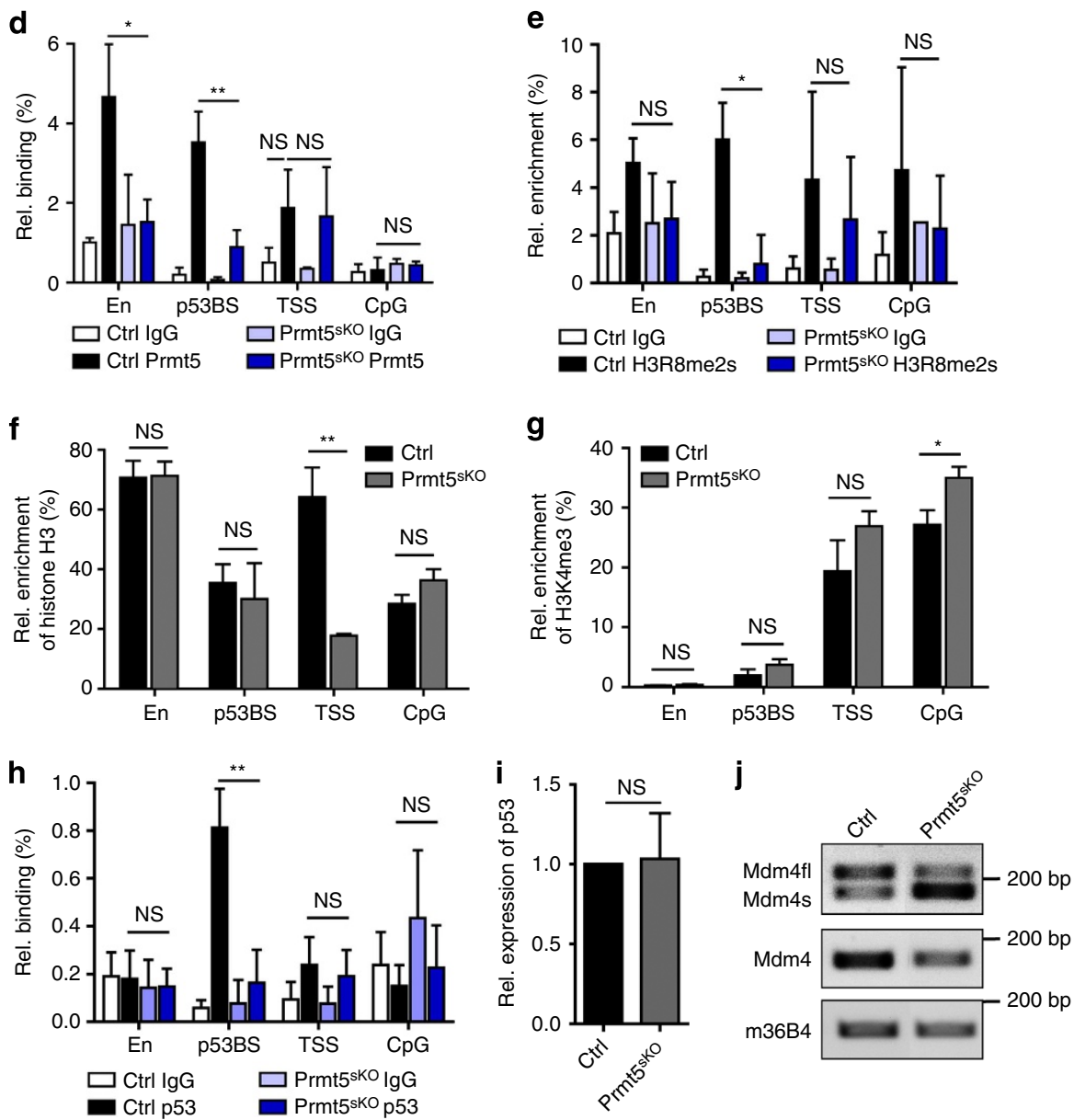

Figure 7 | Epigenetic silencing of the cell cycle inhibitor p21 by Prmt5. (a) RT-qPCR analysis of p21 upregulation in isolated quiescent satellite cells of $\operatorname{Prmt5}^{\text {SKO }}$ mice $(n=4$, each). (b) RT-qPCR analysis of Prmt5, p21, cyclinB1 and myogenic factors after Prmt5 inactivation by addition of 4-OH-TAM ( $n=3$, each). Expression levels of different mRNAs were normalized to GAPDH mRNA. Error bars indicate s.d. of the mean $\left(t\right.$-test: ${ }^{\star} P<0.05$; ${ }^{\star \star} P<0.01$; NS, $P>0.05$ ). (c) Schematic outline of the localization of four important regulatory regions in the p21 gene locus: enhancer-like (En), p53 binding site (p53BS), transcriptional start site (TSS) and intronic $\mathrm{CpG}$ island (CpG). Primer pairs used for ChIP assays are indicated by arrows. The p53 consensus motif of the murine and human p21 gene locus is shown. (d-h) Quantitative PCR analyses of ChIP using antibodies against Prmt5 (d), H3R8me2s (e), histone H3 (f), H3K4me3 (g) and p53 (h) at indicated regulatory regions of the p21 gene locus in MuSC of Prmt5sKO and control mice ( $n=3$, each). Relative (Rel.) enrichment of Prmt5, H3 and p53 was normalized to input DNA. Enrichment of H3R8me2 and H3K4me3 was normalized to histone H3. Error bars represent s.d.'s of the mean ( $t$-test: ${ }^{\star \star} P<0.01$; ${ }^{\star} P<0.05$; NS, $P>0.1$ ). (i) RT-qPCR analysis of p53 mRNA levels in MuSC of Prmt5 ${ }^{5 K O}$ mice and control littermates ( $n=3$, each). Error bars represent s.d.'s of the mean ( $t$-test: NS, $P>0.05$ ). (j) Semi-quantitative RT-PCR analysis of different Mdm4 splicing isoforms in MuSC of Prmt5 ${ }^{5 K O}$ mice and control littermates. RT-PCR using primers in the $3^{\prime}$ untranslated region of Mdm4 detecting both Mdm4fl and Mdm4s was used as loading control. RT-PCR-mediated detection of m36B4 served as an additional loading control. Control (Ctrl): Pax7CreERT2+/-/

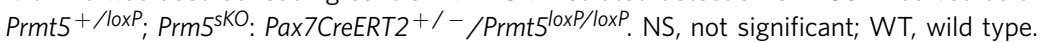


a

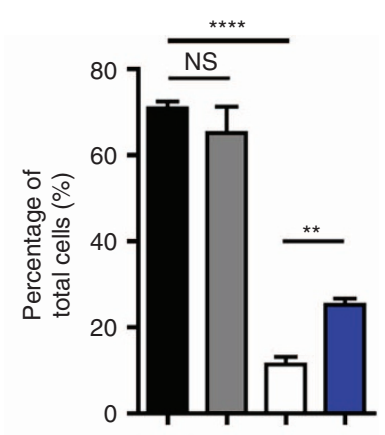

Ctrl $\square$ Prmt5 ${ }^{\text {sKo }}$

$\square \mathrm{p} 21^{-/-} \square$ Prmt5sko/p21-/-

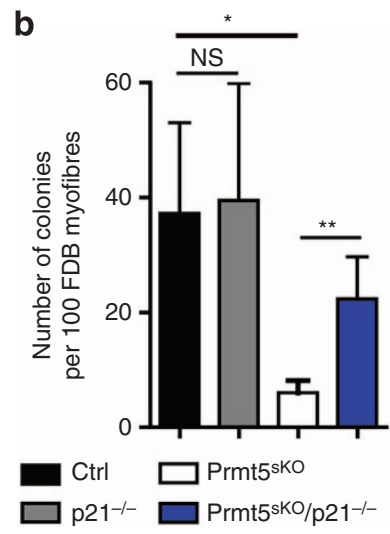

Prmt5sko /p21-/-

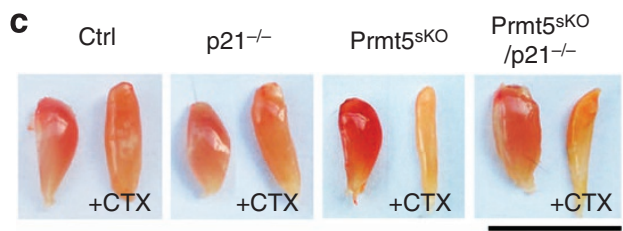

d $\quad \operatorname{Ctrl}$
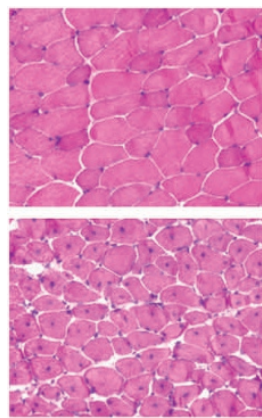

$\mathrm{p} 21^{-/-}$
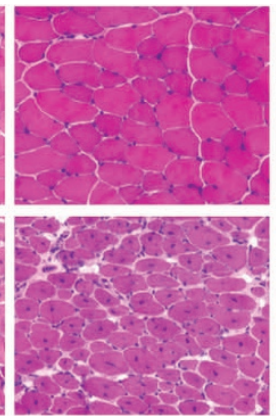

Prmt5sko

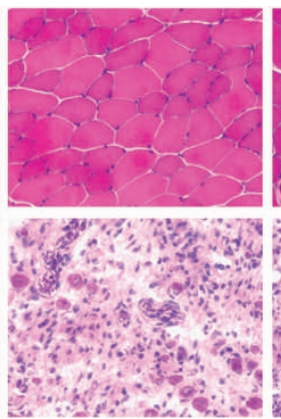

$\operatorname{Prmt5} 5 \mathrm{skO} / \mathrm{p} 21^{-1-}$

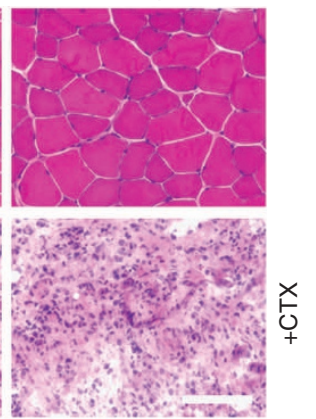

Figure 8 | Prmt5 regulates proliferation of MuSC via inhibition of p21 expression. (a) EdU incorporation in isolated MuSCs reveals a partial rescue of MuSC proliferation in Prmt5 $5^{5 K O} / \mathrm{p} \mathrm{21}^{-/-}$compared with $P r m t 5^{5 K O}$ mice $\left(n=3\right.$, each). Error bars represent s.d.'s of the mean ( $t$-test: ${ }^{\star \star \star \star} P<0.0001$; ${ }^{\star \star} P<0.01$; NS, $P>0.05$ ). (b) Increased numbers of Pax $7^{+}$colonies on $P r m t 5^{s K O} / p 21^{-1}$ - compared with Prmt5 $5^{5 K O} 3$-day cultured myofibres $(n=4$, each). Numbers of $\mathrm{Pax} 7^{+}$colonies from 100 myofibres are shown. Error bars represent s.d.'s of the mean $\left(t\right.$-test: $\left.{ }^{\star \star} P<0.01 ;{ }^{\star} P<0.1 ; N S, P>0.05\right)$.

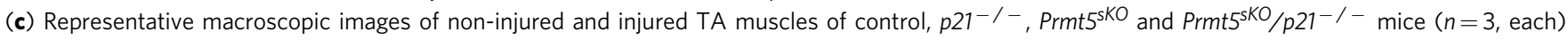
14 days after CTX injection. Scale bar, $1 \mathrm{~cm}$. (d) Haematoxylin and eosin (H\&E) staining of muscle section from non-injured (upper panel) and injured TA (CTX lower panel) muscles of control, $p 21^{-/-}, \operatorname{Prmt} 5^{5 K O}$ and $P r m t 55 K / p 21^{-/-}$mice $(n=3$, each) 14 days after CTX injection. Control (Ctrl):

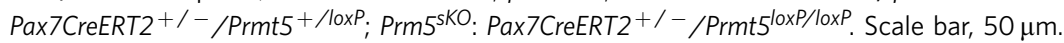

MuSC in muscle dystrophies, analyse their function in muscle hypertrophy or to block proliferation of tumour cells in rhabdomyosarcomas.

\section{Methods}

Animals. The Prmt5 $5^{\text {loxP } / l o x P}$ mouse strain was obtained from EUCOMM.

Rosa2 $6^{\text {nlacZ }}$ and $C 57 B L / 10 S c S n-D m d m d x / J(m d x)$ mouse strain was obtained from The Jackson Laboratory (Bar Harbor, ME). Generation of Pax 7 CreERT2 (ref. 7), Rosa26 $^{Y F P}$ (ref. 39) Pax $7^{I C N}$ (ref. 14), p21 null (ref. 22), Pax $7^{Z s G r e e n}$ (ref. 12) and $Z / E G$ reporter ${ }^{6}$ mice have been described previously. Primers used for genotyping are shown in Supplementary Table 1. TAM (Sigma) was administered intraperitoneally at $3 \mathrm{mg}$ per $40 \mathrm{~g}$ body weight per injection. CTX $\left(0.06 \mathrm{mg} \mathrm{ml}^{-1}\right.$, Sigma) was injected into TA muscles in a volume of $50 \mu$ l. All animal experiments were done in accordance with the Guide for the Care and Use of Laboratory Animals published by the US National Institutes of Health (NIH Publication No. 85-23, revised 1996) and according to the regulations issued by the Committee for Animal Rights Protection of the State of Hessen (Regierungspraesidium Darmstadt).

Myofibre isolation and MuSC purification. The FDB muscles were isolated and digested with $0.2 \%$ collagenase P (Roche) in DMEM medium. The isolated myofibres were either fixed directly with $4 \%$ paraformaldehyde (PFA) or fixed after 3 -day culturing in DMEM medium with $20 \%$ fetal calf serum (FCS) and basic fibroblast growth factor (bFGF) $\left(5 \mathrm{ng} \mathrm{m}^{-1}\right)$. Satellite cell isolation and purification were performed according to established methods ${ }^{6}$. Briefly limb and trunk muscles were minced, digested with $100 \mathrm{CU}$ Dispase (BD) and $0.2 \%$ type II collagenase (Worthington Biochemicals), and consecutively filtered through 100-, 70- and 40$\mu \mathrm{m}$ cell strainers (BD). Cells were applied to a discontinuous Percoll gradient consisting of $70 \%$ Percoll overlayed with $30 \%$ (vol/vol) Percoll. Mononuclear cells were collected at the 70/30 interphase and subjected to FACS (BD FACSAriaII) either using immunostaining with fluorescence-coupled primary antibodies $\left(\mathrm{CD}_{11 b^{-}}, \mathrm{CD}_{45}{ }^{-}, \mathrm{CD}_{3} 1^{-}, \mathrm{CXCR}^{+}{ }^{+}\right.$and $\mathrm{CD} 34^{+}$; refs $\left.6,13,40\right)$ or GFP fluorescence of satellite cells from $\operatorname{Pax}^{Z}{ }_{\text {SGGreen }}$ mice and Pax $7^{\text {CreERT2 }} /$ Rosa $26^{\text {YFP }}$ mice. FACS-purified SCs were cultured on Matrigel-coated 384-well $\mu$ Clear plates (BD Biosciences, Greiner) in DMEM medium with $20 \%$ FCS and bFGF

$\left(5 \mathrm{ng} \mathrm{ml}^{-1}\right.$; refs 6,8). EdU incorporation assay was performed by adding EdU with a final concentration of $10 \mu \mathrm{M} 3 \mathrm{~h}$ before fixation and then analysed using the Click-iT EdU kit (Invitrogen) according to the manufacturer's protocol. TUNEL assays to monitor apoptosis were carried out with the In Situ Cell Death Detection Kit (Roche) according to the manufacturer's protocol. For Cre recombinasemediated in vitro ablation of Prmt5, cultured satellite cells were treated with 4-OH-TAM $(0.4 \mathrm{mM}$, Calbiochem) for 4 days and were analysed 5 days later. Lentiviruses overexpressing the coding region of human Prmt5 were generated with a modified lentiviral vector derived from plko.1 (Sigma-Aldrich) in HEK293T cells using the helper plasmids pMD2.G and psPAX2, and used for infection of MuSC.

Immunofluorescence and morphological analysis. Cultured cells and myofibres were fixed in $4 \%$ PFA. Frozen muscle sections $(5-10 \mu \mathrm{m})$ were fixed in cold acetone. Primary antibodies for immunohistochemical staining are shown in 
a
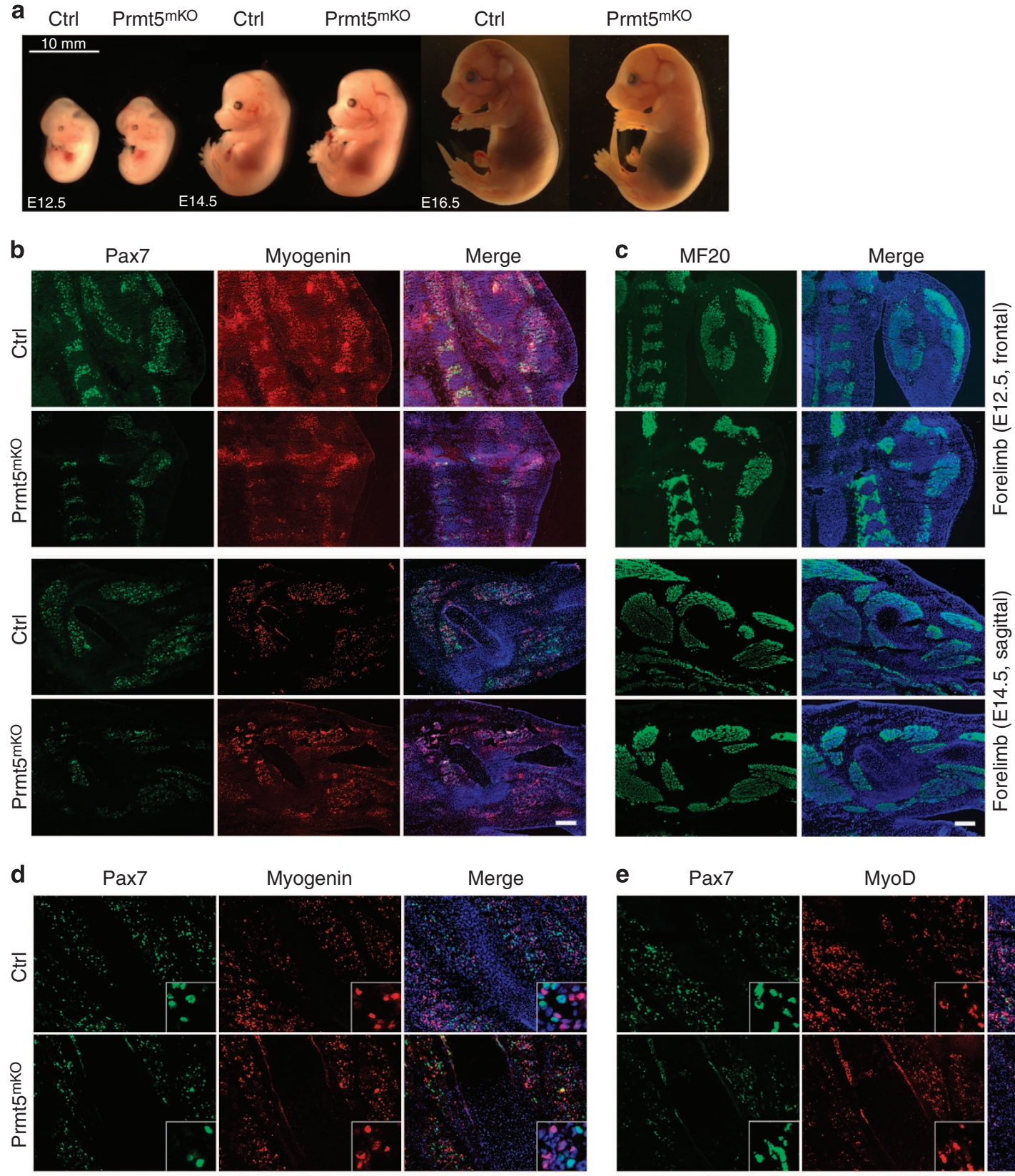

e $\quad \operatorname{Pax} 7$

MyoD

Merge
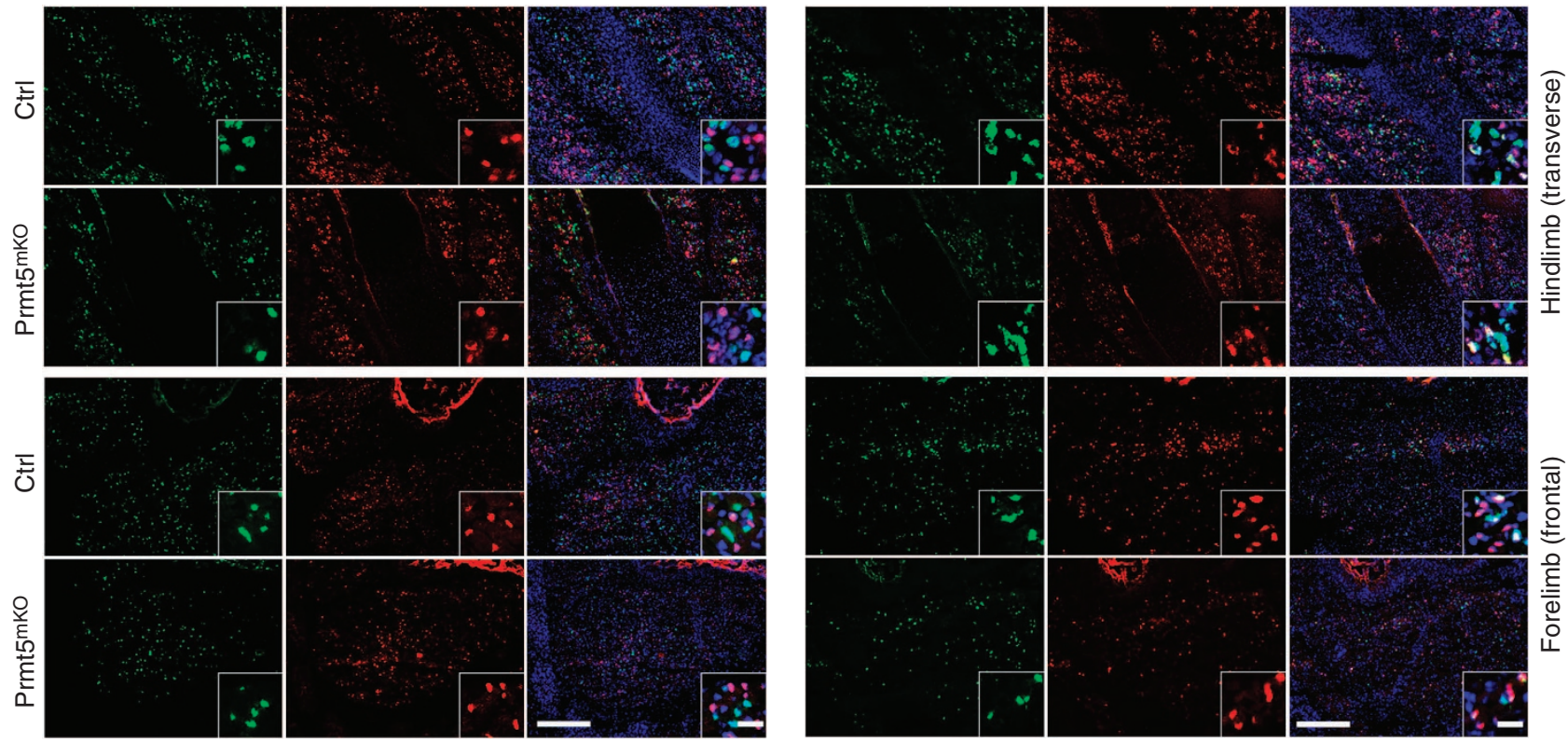

Figure 9 | Prmt5 is dispensable in embryonic Pax7 ${ }^{+}$muscle progenitor cells. (a) Representative images of control and Prmt5 $5^{\mathrm{mKO}}$ embryos at embryonic day E12.5, E14.5 and E16.5. ( $n=3$, each). Scale bar, $10 \mathrm{~mm}$. (b,c) Immunofluorescence images of cryosectioned forelimbs and hindlimbs. (b) E12.5 frontal sections: upper panel; E14.5 sagittal sections: lower panel, Pax7 (green), myogenin (red), DAPI (blue). Scale bar, $50 \mu \mathrm{m}$. (c) E12.5 frontal sections: upper panel; E14.5 sagittal sections: lower panel, MF20 (green), DAPI (blue). Scale bar, $50 \mu \mathrm{m}$. (d,e) Immunofluorescence of cryosections from hindlimbs. E16.5 transverse sections: upper panel and forelimbs. E16.5 frontal sections: lower panel, Pax7 (green), myogenin (red; d), and Pax7 (green) and MyoD

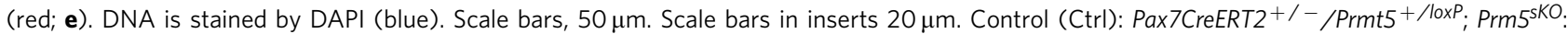
Pax7CreERT2 $+1-/$ Prmt5 ${ }^{\text {loxP/loxP. }}$. 


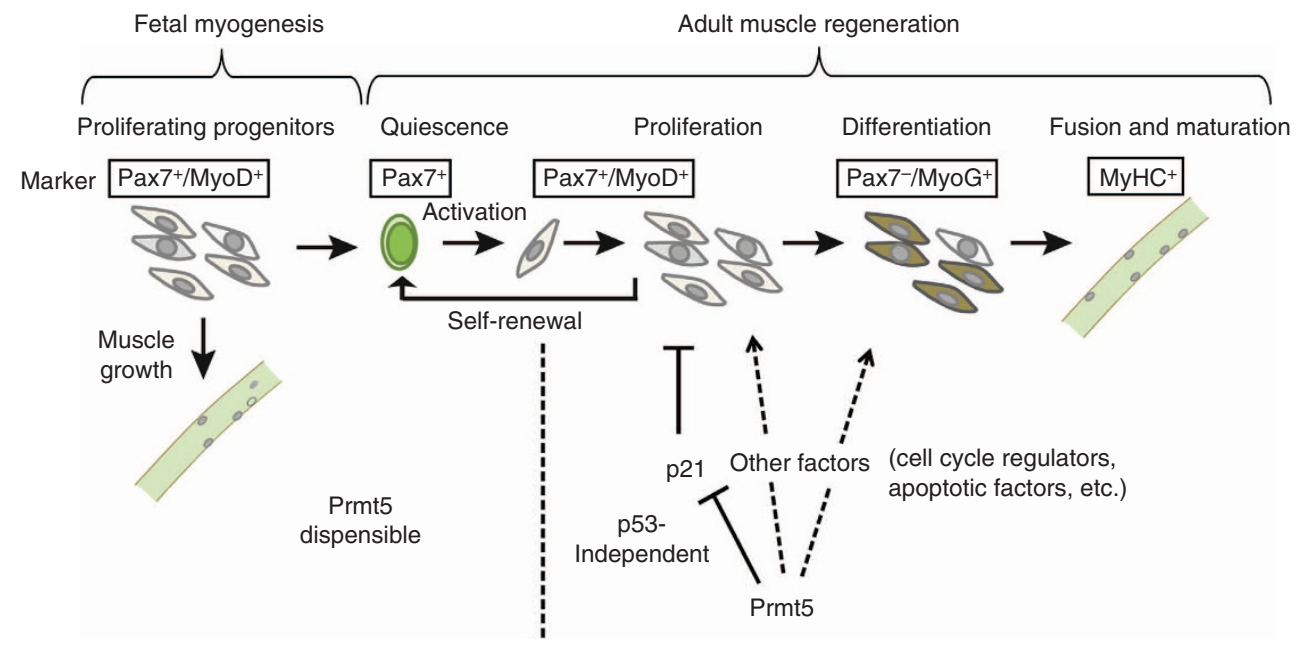

Figure 10 | Model of the role of Prmt5 during fetal myogenesis and adult muscle regeneration. Prmt5 controls proliferation of adult MuSC by direct epigenetic silencing of the cell cycle inhibitor $p 21$ independent of p53, but is dispensable for proliferation and differentiation of Pax ${ }^{+}$muscle progenitor cells during fetal myogenesis. Prmt5 does not affect the initial activation of MuSC resulting in MyoD expression but enables proliferation of MuSC, thus generating a poised state, which keeps MuSC in a standby mode allowing rapid MuSC amplification under disease conditions. Inactivation of Prmt5 in proliferating MuSC does not suppress MyoG expression but blocks differentiation, thereby suggesting an additional function of Prmt5 for muscle cell differentiation. Proliferating fetal muscle progenitor cells are defined by expression of Pax7 and MyoD, quiescent MuSC by expression of Pax7 and lack of MyoD expression, proliferating MuSC by the concomitant expression of Pax7 and MyoD, and differentiating muscle cells by expression of MyoG. Differentiated muscle cells are marked by expression of myosin heavy chain (MyHC).

Supplementary Table 2. LacZ staining of regenerating or normal TA muscle sections 3 days after CTX injection was carried out according to standard protocols, Eosin staining was used to visualize muscle fibres. Masson's Trichrome staining was carried out using the ACCUSTAIN trichrome staining kit (Sigma-Aldrich) following the manufacturer's instructions.

ChIP and RT-qPCR. ChIP assays on purified satellite cells were performed according to established protocols modified for small cell numbers ${ }^{41}$. FACSpurified satellite cells $(100,000)$ were first crosslinked with $1 \%$ formaldehyde for $10 \mathrm{~min}$ and then quenched by $0.125 \mathrm{M}$ glycine for $15 \mathrm{~min}$ at room temperature. Chromatin was sheared to lengths of $300-500$ bp using Bioruptor (Diagenode) and subjected to immunoprecipitation with antibodies are shown in Supplementary Table 2. Primers used for ChIP-qPCR are shown in Supplementary Table 3. For RT-qPCR assays, total RNA from satellite cells and muscles was isolated using Trizol reagent (Invitrogen) according to the manufacturer's protocol. An amount of $1 \mu \mathrm{g}$ of purified RNA was subjected to reverse transcriptase reaction in the presence of $25 \mathrm{ng} \mathrm{ml}^{-1}$ random primers and $2.5 \mathrm{mM} \mathrm{dA} / \mathrm{C} / \mathrm{G} / \mathrm{TTP}$ with $10 \mathrm{U} \mathrm{ml}^{-1}$ SuperScript II Reverse Transcriptase (Invitrogen). Primers used for RT-qPCR are shown in Supplementary Table 4.

Western blot analysis. For western blot assays in vitro cultured satellite cells were harvested, washed with ice-cold PBS and lysed in cell lysis buffer $(20 \mathrm{mM}$ Tris (pH 7.5), $400 \mathrm{mM} \mathrm{NaCl}, 1 \mathrm{mM}$ EDTA, 1 mM EGTA, 1\% Triton X-100, $2.5 \mathrm{mM}$ sodium pyrophosphate, $1 \mathrm{mM} \beta$-glycerophosphate, $1 \mathrm{mM} \mathrm{Na}_{3} \mathrm{VO}_{4}$ and $1 \mu \mathrm{g} \mathrm{ml} \mathrm{m}^{-1}$ leupeptin). Whole-cell lysates $(10 \mu \mathrm{g})$ were subjected to SDS-PAGE and western blotting using antibodies are shown in Supplementary Table 2. Protein expression was visualized using an enhanced chemiluminescence detection system (GE Healthcare, Little Chalfont, UK) and quantified using a ChemiDoc gel documentation system (Bio-Rad). Uncropped images of original western blots are presented in (Supplementary Fig. 4).

RNA-seq. For RNA-seq, RNA was isolated from cultured satellite cells using the miRNeasy micro Kit (Qiagen) combined with on-column DNase digestion (DNase-Free DNase Set, Qiagen) to avoid contamination by genomic DNA. RNA and library preparation integrity were verified with a BioAnalyzer (Agilent). Ribosomal depletion was performed using RiboMinus Eukaryote System v2 (Life Technologies) with $500 \mathrm{ng}$ total RNA as input following the low input protocol. Libraries were prepared with the Ion Total RNA-Seq Kit v2 (Life Technologies) adjusting the total amount of depleted and fragmented RNA to standard protocols. Sequencing was performed on the Ion Torrent Proton platform using V3 chemistry (Ion PI Template OT2 200 Kit v3, Life Technologies) and PIV2 Chips (Ion PI Chip Kit v2, Life Technologies). For each run, two RNA-seq libraries were measured resulting in $82 \mathrm{M}$ reads in total and at least $40 \mathrm{M}$ reads per library. Raw reads were assessed for quality, adaptor content and duplication rates with FastQC 0.10.1, trimmed by Reaper version 13-100 (ref. 42) and terminally aligned to the Ensemble mouse genome version mm10 (GRCm38) by STAR 2.4.0a (ref. 43). Differentially expressed genes were identified using DESeq2 version 1.62 (ref. 44). Only genes with a minimum fold change of \pm 2 , a maximum Benjamini-Hochberg corrected $P$ value of 0.05 and a minimum combined mean of 5 reads were classified as significantly differentially expressed. The Ensemble annotation was enriched with UniProt data (release 06.06.2014) based on Ensemble gene identifiers. Gene ontology analysis was performed using DAVID Bioinformatics Resources (http://david.abcc.ncifcrf.gov) ${ }^{45}$. Heatmaps of cell cycle-related genes were generated using 'heatmap.2' in R 'gplots' package. Gene expression data were deposited in the NCBI Geo database (http://www.ncbi.nlm.nih.gov/geo/query/ acc.cgi?acc=GSE66822) under the accession number GSE66822.

Mass spectrometry. Muscle and satellite cell samples were homogenized in lysis buffer (4\% SDS in $100 \mathrm{~mm}$ Tris/HCl, $\mathrm{pH}$ 7.6). Lysates were loaded on an SDS-PAGE (NuPAGE 4-12\% BisTris gel, Invitrogen) and stained with colloidal Protein Staining Solution (Invitrogen). Evenly sized gel pieces were excised for in-gel digestion using trypsin after reduction and alkylation of gel pieces. Gel pieces were washed twice with $50 \%\left(50 \mathrm{mM} \mathrm{NH}_{4} \mathrm{HCO}_{3} / 50 \%\right.$ ethanol) for $20 \mathrm{~min}$, dehydrated with $100 \%$ ethanol for $10 \mathrm{~min}$ and vacuum dried. Gel pieces were reduced with $10 \mathrm{mM}$ dithiothreitol for $45 \mathrm{~min}$ at $56^{\circ} \mathrm{C}$ and alkylated with $55 \mathrm{mM}$ iodocetamide (BioUltra, Sigma-Aldrich) for $30 \mathrm{~min}$ at room temperature in the dark. After two steps of washing/dehydration, samples were dehydrated twice with $100 \%$ ethanol for $15 \mathrm{~min}$ and vacuum dried. Gel pieces were digested overnight at $37^{\circ} \mathrm{C}$ in $50 \mu \mathrm{l}$ of digestion buffer containing $12.5 \mathrm{ng} \mu \mathrm{l}$ of Sequencing Grade Modified Trypsin (Promega Corp., Madison, USA). Released peptides were extracted (collecting separately the liquid mixture of each samples at each step) once by adding $100 \mu \mathrm{l}$ of $30 \%$ acetonitrile liquid chromatography/mass spectrometry (LC/MS) grade (Thermo Scientific)/3\% trifluoroacetic acid (Sigma-Aldrich) in water, and twice by adding $70 \%$ acetonitrile, followed by two final extractions with $100 \%$ acetonitrile. Extracts were vacuum dried to remove acetonitrile and subsequently acidified with $0.5 \%$ trifluoroacetic acid (TFA) ${ }^{46}$. Peptides were purified by stop and go extraction tips ${ }^{47}$, and were run on a LC system coupled to a LTQ-Orbitrap XL or a LTQ-Orbitrap Velos mass spectrometer (Thermo Fisher Scientific) equipped with a nanoelectrospray source (Proxeon). Full-survey scan spectra $(m / z=300-1,650)$ were acquired in the Orbitrap with a resolution of $R=60,000$ after accumulation of $1,000,000$ ions. Raw data were analysed using the MaxQuant software package ${ }^{48}$. Database searches were performed with the Mascot search engine against a murine FASTA database (IPI 3.54). A false discovery rate of $1 \%$ was used, and only peptides with a minimum of six amino-acid length with at least one unique peptide were included for data analysis.

High-throughput screen and hit validation. A customized shRNA library against selected genes was robotically re-arrayed from a murine genome-wide The RNAi Consortium (TRC) shRNA library ${ }^{49}$ (Sigma-Aldrich). Plasmids were purified with 
GenElute HP 96-Well Miniprep Kits (Sigma-Aldrich) and tested for integrity with a PvuII digest. High-throughput production of lentivirus was performed by $\mathrm{Ca}_{3}\left(\mathrm{PO}_{4}\right)_{2}$ transfection of HEK293T cells with helper plasmids pMD2.G and psPAX2. Four-hundred fifty freshly isolated satellite cells per well were seeded into 384-well tissue culture plates freshly coated with Matrigel (Greiner). Twenty-four hours later, cells were transduced with lentiviral supernatants supplemented with $8 \mu \mathrm{g} \mathrm{ml}^{-1}$ polybrene for $6 \mathrm{~h}$. After media exchange, cells were incubated for $72 \mathrm{~h}$. Each 384-well plate contained 56 controls including 12 individual GFP-producing lentiviruses to monitor transduction efficiency, four positive controls for Pax7 knockdown (shRNA Pax7) and four positive controls for Nf1 knockdown (shRNA Nf1). Cells were fixed in 4\% PFA and whole-well images were acquired and analysed using an ImageXpress Micro automated high-throughput fluorescence microscope and MetaXpress software (Molecular Devices). Pax7/DAPI ratios were determined for each individual well. Only plates with $Z^{\prime}$ values $>0.5$ according to $1-\left(2 \sigma_{\text {pos }}+2 \sigma_{\text {neg }} / \mu_{\text {neg }}-\mu_{\text {pos }}\right)$ were used for further processing. Values as reference for Pax7 expression in percentage were then calculated according to $z=(x / \mu)(-1)(x$, value of particular sample; $\mu$, mean of plkol empty vector; $n=4)$. Target genes qualified as hits if percentiles were higher or lower than $25 \%$ compared with control.

Magnetic resonance imaging. All MRI experiments were performed on a 7.0-T superconducting magnet (Bruker Biospin, Pharmascan, 70/16, $16 \mathrm{~cm}$; Ettlingen, Germany) equipped with an actively shielded imaging gradient field of $300 \mathrm{mT} \mathrm{m}^{-1}$ (ref. 50). The frequency for the ${ }^{1} \mathrm{H}$ isotope is $300.33 \mathrm{MHz}$. A $60-\mathrm{mm}$ inner diameter linear-polarized ${ }^{1} \mathrm{H}$ volume resonator was used for RF pulse transmission and signal reception (Bruker Biospin). Localized images were acquired using a spin-echo sequence and corrections of slice angulation were performed, if necessary. RARE (Rapid Acquisition with Relaxation Enhancement) sequences (repetition time $(\mathrm{TR})=2,500 \mathrm{~ms}$, echo time $(\mathrm{TE})=36.7 \mathrm{~ms}$, slice thickness $=1 \mathrm{~mm}$ ) in axial and coronal orientation were used to determine exact positioning of the lower part of the mouse body. A coronal MSME (Multi-SliceMulti-Echo)-spin-echo-sequence with an echo time $\mathrm{TE}=8.6 \mathrm{~ms}$, repetition time $\mathrm{TR}=453 \mathrm{~ms}$, a field of view FOV $=7 \times 7 \mathrm{~cm}^{2}$, matrix size MTX $=512 \times 256$ and a slice thickness of $1 \mathrm{~mm}$ was recorded. Volumetric quantification of fat and muscle tissue from images was processed by software ImageJ. A list of anatomically defined landmarks was used to derive tissue-specific signal intensity thresholds and to define the region of interest for intensity sensitive region growing segmentation. The resulting tissue voxel volumes inside the region of interest were determined as cubic millimetres for each tissue class. Mice were measured under volatile isoflurane (1.5-2.0\% in oxygen and air with a flow rate of $\left.1.01 \mathrm{~min}^{-1}\right)$ anaesthesia; the body temperature was maintained at $37^{\circ} \mathrm{C}$ by a thermostatically regulated water flow system during the entire imaging protocol.

Statistics. For statistical analysis, the two following tests (two-tailed) were used: (1) three or more groups: one factorial ANOVA; (2) two groups: unpaired $t$-test. $P$ values $<0.05$ were considered statistically significant. Data were analysed using GraphPad Prism v5.03 (GraphPad Software, San Diego, CA).

\section{References}

1. Wilson, A. et al. Hematopoietic stem cells reversibly switch from dormancy to self-renewal during homeostasis and repair. Cell 135, 1118-1129 (2008).

2. Brack, A. S. \& Rando, T. A. Tissue-specific stem cells: lessons from the skeletal muscle satellite cell. Cell Stem Cell 10, 504-514 (2012).

3. Schienda, J. et al. Somitic origin of limb muscle satellite and side population cells. Proc. Natl Acad. Sci. USA 103, 945-950 (2006).

4. Seale, P. et al. Pax7 is required for the specification of myogenic satellite cells. Cell 102, 777-786 (2000).

5. Relaix, F., Rocancourt, D., Mansouri, A. \& Buckingham, M. A Pax3/Pax7dependent population of skeletal muscle progenitor cells. Nature 435, 948-953 (2005).

6. Gunther, S. et al. Myf5-positive satellite cells contribute to pax7-dependent long-term maintenance of adult muscle stem cells. Cell Stem Cell 13, 590-601 (2013).

7. Lepper, C., Conway, S. J. \& Fan, C. M. Adult satellite cells and embryonic muscle progenitors have distinct genetic requirements. Nature 460, 627-631 (2009).

8. Oustanina, S., Hause, G. \& Braun, T. Pax7 directs postnatal renewal and propagation of myogenic satellite cells but not their specification. EMBO J. 23, 3430-3439 (2004)

9. Kuang, S., Kuroda, K., Le Grand, F. \& Rudnicki, M. A. Asymmetric self-renewal and commitment of satellite stem cells in muscle. Cell 129, 999-1010 (2007).

10. Yin, H., Price, F. \& Rudnicki, M. A. Satellite cells and the muscle stem cell niche. Physiol. Rev. 93, 23-67 (2013).

11. Fukada, S. et al. Isolation, characterization, and molecular regulation of muscle stem cells. Front. Physiol. 4, 317 (2013).

12. Bosnakovski, D. et al. Prospective isolation of skeletal muscle stem cells with a Pax7 reporter. Stem Cells 26, 3194-3204 (2008).
13. Montarras, D. et al. Direct isolation of satellite cells for skeletal muscle regeneration. Science 309, 2064-2067 (2005).

14. Keller, C., Hansen, M. S., Coffin, C. M. \& Capecchi, M. R. Pax3:Fkhr interferes with embryonic Pax3 and Pax7 function: implications for alveolar rhabdomyosarcoma cell of origin. Genes Dev. 18, 2608-2613 (2004).

15. Conboy, M. J., Cerletti, M., Wagers, A. J. \& Conboy, I. M. Immuno-analysis and FACS sorting of adult muscle fiber-associated stem/precursor cells. Methods Mol. Biol. 621, 165-173 (2010).

16. Bedford, M. T. \& Richard, S. Arginine methylation an emerging regulator of protein function. Mol. Cell 18, 263-272 (2005).

17. Tee, W. W. et al. Prmt5 is essential for early mouse development and acts in the cytoplasm to maintain ES cell pluripotency. Gene Dev. 24, 2772-2777 (2010).

18. Gkountela, S., Li, Z., Chin, C. J., Lee, S. A. \& Clark, A. T. PRMT5 is required for human embryonic stem cell proliferation but not pluripotency. Stem Cell Rev. 10, 230-239 (2014).

19. Bezzi, M. et al. Regulation of constitutive and alternative splicing by PRMT5 reveals a role for Mdm4 pre-mRNA in sensing defects in the spliceosomal machinery. Gene Dev. 27, 1903-1916 (2013).

20. Sacco, A. et al. Short telomeres and stem cell exhaustion model Duchenne muscular dystrophy in mdx/mTR mice. Cell 143, 1059-1071 (2010).

21. Dacwag, C. S., Ohkawa, Y., Pal, S., Sif, S. \& Imbalzano, A. N. The protein arginine methyltransferase Prmt5 is required for myogenesis because it facilitates ATP-dependent chromatin remodeling. Mol. Cell. Biol. 27, 384-394 (2007).

22. Choudhury, A. R. et al. Cdknla deletion improves stem cell function and lifespan of mice with dysfunctional telomeres without accelerating cancer formation. Nat. Genet. 39, 99-105 (2007).

23. Spencer, S. L. et al. The proliferation-quiescence decision is controlled by a bifurcation in CDK2 activity at mitotic exit. Cell 155, 369-383 (2013).

24. Parker, S. B. et al. p53-independent expression of p21Cip1 in muscle and other terminally differentiating cells. Science 267, 1024-1027 (1995).

25. Saramaki, A., Banwell, C. M., Campbell, M. J. \& Carlberg, C. Regulation of the human p21(waf1/cip1) gene promoter via multiple binding sites for p53 and the vitamin D3 receptor. Nucleic Acids Res. 34, 543-554 (2006).

26. Siatecka, M., Lohmann, F., Bao, S. \& Bieker, J. J. EKLF directly activates the p21WAF1/CIP1 gene by proximal promoter and novel intronic regulatory regions during erythroid differentiation. Mol. Cell. Biol. 30, 2811-2822 (2010).

27. Batut, J., Duboe, C. \& Vandel, L. The methyltransferases PRMT4/CARM1 and PRMT5 control differentially myogenesis in zebrafish. PloS ONE 6, e25427 (2011).

28. Hutcheson, D. A., Zhao, J., Merrell, A., Haldar, M. \& Kardon, G. Embryonic and fetal limb myogenic cells are derived from developmentally distinct progenitors and have different requirements for beta-catenin. Genes Dev. 23, 997-1013 (2009).

29. Ge, X. et al. Smad3 signaling is required for satellite cell function and myogenic differentiation of myoblasts. Cell Res. 21, 1591-1604 (2011).

30. Cornelison, D. D. et al. Essential and separable roles for Syndecan-3 and Syndecan-4 in skeletal muscle development and regeneration. Genes Dev. 18, 2231-2236 (2004).

31. McKinnell, I. W. et al. Pax7 activates myogenic genes by recruitment of a histone methyltransferase complex. Nat. Cell Biol. 10, 77-84 (2008).

32. Kawabe, Y., Wang, Y. X., McKinnell, I. W., Bedford, M. T. \& Rudnicki, M. A. Carm1 regulates Pax7 transcriptional activity through MLL1/2 recruitment during asymmetric satellite stem cell divisions. Cell Stem Cell 11, 333-345 (2012).

33. Wang, T., Gu, S., Ronni, T., Du, Y. C. \& Chen, X. In vivo dual-tagging proteomic approach in studying signaling pathways in immune response. J. Proteome Res. 4, 941-949 (2005).

34. Kanade, S. R. \& Eckert, R. L. Protein arginine methyltransferase 5 (PRMT5) signaling suppresses protein kinase Cdelta- and p38delta-dependent signaling and keratinocyte differentiation. J. Biol. Chem. 287, 7313-7323 (2012).

35. Braun, T. \& Gautel, M. Transcriptional mechanisms regulating skeletal muscle differentiation, growth and homeostasis. Nat. Rev. Mol. Cell Biol. 12, 349-361 (2011).

36. Shi, X. \& Garry, D. J. Muscle stem cells in development, regeneration, and disease. Genes Dev. 20, 1692-1708 (2006).

37. Buckingham, M. \& Rigby, P. W. Gene regulatory networks and transcriptional mechanisms that control myogenesis. Dev. Cell 28, 225-238 (2014).

38. Dacwag, C. S., Bedford, M. T., Sif, S. \& Imbalzano, A. N. Distinct protein arginine methyltransferases promote ATP-dependent chromatin remodeling function at different stages of skeletal muscle differentiation. Mol. Cell. Biol. 29, 1909-1921 (2009).

39. Srinivas, S. et al. Cre reporter strains produced by targeted insertion of EYFP and ECFP into the ROSA26 locus. BMC Dev. Biol. 1, 4 (2001).

40. Sherwood, R. I. et al. Isolation of adult mouse myogenic progenitors: functional heterogeneity of cells within and engrafting skeletal muscle. Cell 119, 543-554 (2004). 
41. Dahl, J. A., Reiner, A. H. \& Collas, P. Fast genomic muChIP-chip from 1,000 cells. Genome Biol. 10, R13 (2009).

42. Davis, M. P., van Dongen, S., Abreu-Goodger, C., Bartonicek, N. \& Enright, A. J. Kraken: a set of tools for quality control and analysis of high-throughput sequence data. Methods 63, 41-49 (2013).

43. Dobin, A. et al. STAR: ultrafast universal RNA-seq aligner. Bioinformatics 29, 15-21 (2013).

44. Love, M. I., Huber, W. \& Anders, S. Moderated estimation of fold change and dispersion for RNA-seq data with DESeq2. Genome Biol. 15, 550 (2014).

45. Dennis, Jr. G. et al. DAVID: database for annotation, visualization, and integrated discovery. Genome Biol. 4, P3, 2003).

46. Shevchenko, A., Tomas, H., Havlis, J., Olsen, J. V. \& Mann, M. In-gel digestion for mass spectrometric characterization of proteins and proteomes. Nat. Protoc. 1, 2856-2860 (2006)

47. Rappsilber, J., Ishihama, Y. \& Mann, M. Stop and go extraction tips for matrixassisted laser desorption/ionization, nanoelectrospray, and LC/MS sample pretreatment in proteomics. Anal. Chem. 75, 663-670 (2003).

48. Cox, J. et al. A practical guide to the MaxQuant computational platform for SILAC-based quantitative proteomics. Nat. Protoc. 4, 698-705 (2009).

49. Moffat, J. et al. A lentiviral RNAi library for human and mouse genes applied to an arrayed viral high-content screen. Cell 124, 1283-1298 (2006).

50. Biesemann, N. et al. Myostatin regulates energy homeostasis in the heart and prevents heart failure. Circ. Res. 115, 296-310 (2014).

51. Kossler, N. et al. Neurofibromin (Nf1) is required for skeletal muscle development. Hum. Mol. Genet. 20, 2697-2709 (2011).

\section{Acknowledgements}

This work was supported by the DFG (Excellence Cluster Cardio-Pulmonary System and SFB TRR81), the LOEWE Center for Cell and Gene Therapy, the German Center for Cardiovascular Research, and the Universities of Giessen and Marburg Lung Center.

We thank Dr Astrid Wietelmann for assistance with MRI analysis and Dr Marc Bickle for help with generating the custom-arrayed lentiviral shRNA library.

\section{Author contributions}

T.Z. performed the research and analysed the data; T.Z., Y.Z. and J.K. designed and performed experiments, analysed the data and wrote the manuscript together with T.B.; S.G. assisted in the design of the experiments and performed experiments; M.K. provided the mass spectrometry analysis; M.L. and C.K. performed the bioinformatics analysis and analysed RNA-sequencing data; J.K. and T.B. conceived and supervised the project together with Y.Z.; and T.B. edited the final version of the manuscript.

\section{Additional information}

Accession number in NCBI Geo database (http://www.ncbi.nlm.nih.gov/geo): GSE66822.

Supplementary Information accompanies this paper at http://www.nature.com/ naturecommunications

Competing financial interests: The authors declare no competing financial interests.

Reprints and permission information is available online at http://npg.nature.com/ reprintsandpermissions/

How to cite this article: Zhang, T. et al. Prmt5 is a regulator of muscle stem cell expansion in adult mice. Nat. Commun. 6:7140 doi: 10.1038/ncomms8140 (2015).

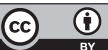
article are included in the article's Creative Commons license, unless indicated otherwise in the credit line; if the material is not included under the Creative Commons license, users will need to obtain permission from the license holder to reproduce the material. To view a copy of this license, visit http://creativecommons.org/licenses/by/4.0/ 